\title{
New Modifications of Integral Inequalities via $\wp$-Convexity Pertaining to Fractional Calculus and Their Applications
}

\author{
Saima Rashid ${ }^{1,+}{ }^{+}$, Aasma Khalid ${ }^{2,+}$, Omar Bazighifan ${ }^{3,4,+}+\left(\right.$ ) and Georgia Irina Oros ${ }^{5, *,+}$ \\ 1 Department of Mathematics, Government College University, Faisalabad 38000, Pakistan; \\ saimarashid@gcuf.edu.pk \\ 2 Department of Mathematics, Government College Women University, Faisalabad 38000, Pakistan; \\ asmakhalid@gcwuf.edu.pk \\ 3 Section of Mathematics, International Telematic University Uninettuno, Corso Vittorio Emanuele II, 39, \\ 00186 Roma, Italy; o.bazighifan@gmail.com \\ 4 Department of Mathematics, Faculty of Science, Hadhramout University, Hadhramout 50512, Yemen \\ 5 Department of Mathematics and Computer Science, University of Oradea, 410087 Oradea, Romania \\ * Correspondence: georgia_oros_ro@yahoo.co.uk \\ + These authors contributed equally to this work.
}

Citation: Rashid, S.; Khalid, A.; Bazighifan, O.; Oros, G.I. New

Modifications of Integral Inequalities

via $\wp$-Convexity Pertaining to

Fractional Calculus and Their Applications. Mathematics 2021, 9, 1753. https://doi.org/10.3390/ math9151753

Academic Editor: Gabriel Eduard Vilcu

Received: 17 June 2021

Accepted: 22 July 2021

Published: 26 July 2021

Publisher's Note: MDPI stays neutral with regard to jurisdictional claims in published maps and institutional affiliations.

Copyright: (c) 2021 by the authors. Licensee MDPI, Basel, Switzerland. This article is an open access article distributed under the terms and conditions of the Creative Commons Attribution (CC BY) license (https:/ / creativecommons.org/licenses/by/ $4.0 /)$.

\begin{abstract}
Integral inequalities for $\wp$-convex functions are established by using a generalised fractional integral operator based on Raina's function. Hermite-Hadamard type inequality is presented for $\wp$-convex functions via generalised fractional integral operator. A novel parameterized auxiliary identity involving generalised fractional integral is proposed for differentiable mappings. By using auxiliary identity, we derive several Ostrowski type inequalities for functions whose absolute values are $\wp$-convex mappings. It is presented that the obtained outcomes exhibit classical convex and harmonically convex functions which have been verified using Riemann-Liouville fractional integral. Several generalisations and special cases are carried out to verify the robustness and efficiency of the suggested scheme in matrices and Fox-Wright generalised hypergeometric functions.
\end{abstract}

Keywords: Hermite-Hadamard inequality; Ostrowski type inequality; $\wp$-convex function; generalised fractional integral; matrices; Fox-Wright function

\section{Introduction}

A new calculus has been revolutionised with integrals and derivatives of arbitrary order. Recently, several researchers introduced a bulk of novel fractional operators which have made a significant contribution to the extension of fractional calculus. In real life, fractional calculus is generated from various fractional operators such as Riemann-Liouville, Caputo, Hadamard, Atangana-Baleanu, Caputo-Fabrizio, Gauss hypergeometric and so on, due to its widespread use in different fields, for example, turbulence, electric networks, exothermic chemical reactions or autocatalytic reactions, modelling, flow of fractional Maxwell fluid and engineering, see [1-3]. Going in the same direction in the setting of fractional operators, fractional differential equations have played a dominant role and investigated useful results in modelling of several phenomena in biological systems with memory and computer graphics [4-9].

As is well known, integral inequalities, which are based on fractional calculus, are widely used in many real-life phenomena, such as coding theory, functional analysis, and optimisation theory. To promote the investigation of fractional integral operator, here, we demonstrate the concept which is extensively utilised for the development of inequalities, namely the generalised fractional integral operator based on Raina's function along with the well-acknowledged concept of convexity that plays a vital role in operation research, economics, fuzzy analysis and management sciences. 
Now, we recall the celebrated Hermite-Hadamard inequality as follows:

$$
F\left(\frac{e_{1}+e_{2}}{2}\right) \leq \frac{1}{e_{2}-e_{1}} \int_{e_{1}}^{e_{2}} F(x) d x \leq \frac{F\left(e_{1}\right)+F\left(e_{2}\right)}{2}
$$

holds for all $e_{1}, e_{2} \in \mathbb{I}$ and $e_{1}<e_{2}$.

Several refinements, improvements and variant forms of (1) have been contemplated in the literature (see, e.g., [10-15]).

Another distinguished generalisation in inequality theory proposed in 1928, is the Ostrowski inequality [16], which provides an upper bound for the approximation of the integral average $\frac{1}{e_{2}-e_{1}} \int_{e_{1}}^{e_{2}} F(\ell) d \ell$ by the value $F(x)$ at $x \in\left[e_{1}, e_{2}\right]$, can be described as follows

$$
\left|\left(e_{2}-e_{1}\right) F(x)-\int_{e_{1}}^{e_{2}} F(\ell) d \ell\right| \leq \mathcal{M}\left(e_{2}-e_{1}\right)^{2}\left[\frac{1}{4}+\frac{\left(x-\frac{e_{1}+e_{2}}{2}\right)^{2}}{\left(e_{2}-e_{1}\right)^{2}}\right]
$$

holds for all $x \in\left(e_{1}, e_{2}\right)$ with the best feasible constant $\frac{1}{4}$.

The inequality (2) has a significant contribution in quadrature rules, numerical analysis and certain special areas of pure and applied sciences. An enormous heft of developments and speculations of (1) and (2) have been established with the aid of fractional operators [17-22].

Here, we intend to derive a refinement of Hermite-Hadamard type integral inequality by the use of generalised fractional integral operator. Taking into account the generalised fractional integral operators, we also obtained an integral identity and more generalised fractional integral inequalities of the Ostrowski type with respect to operators (4) and (5). We now define some basic concepts, preliminaries, definitions and related consequences.

Definition 1. We call the mapping $F: \varnothing \neq \mathbb{K} \subseteq \mathbb{R} \rightarrow \mathbb{R}$ is convex on $\mathbb{K}$ if

$$
F(\ell x+(1-\ell) y) \leq \ell F(x)+(1-\ell) F(y), \forall x, y \in \mathbb{K}, \ell \in[0,1] .
$$

Definition 2 ([23]). Let $\wp \in \mathbb{R} \backslash\{0\}$ and $\mathbb{K} \subseteq(0, \infty)$. We call the mapping $F: \mathbb{K} \subseteq \mathbb{R} \rightarrow \mathbb{R}$ is $\wp$-convex on $\mathbb{K}$ if

$$
F\left(\left[\ell x^{\wp}+(1-\ell) y^{\wp}\right]^{\frac{1}{\wp}}\right) \leq \ell F(x)+(1-\ell) F(y), \forall x, y \in \mathbb{K}, \ell \in[0,1] .
$$

A lot of researchers have been expanding $\wp$-convex functions and their characteristics. For example, Abdeljawad et al. [24] derived Simpson's type inequalities for $\wp$-convex functions on fractal space. Chen et al. [25] explored the fractional approach for $n$-polynomial $\wp$-convex functions. İşcan et al. [26] obtained some Hermite-Hadamard inequality for $\wp$-quasi convex functions. More detailed implications for $\wp$-convex functions can be found in the works $[13,23,27]$.

Now, we recall the generalised fractional integral operators, which are necessary for our main results.

Raina [28] introduced the following operator associated with the general class of functions.

$$
\mathcal{F}_{\rho, \varphi}^{\sigma}(x)=\mathcal{F}_{\rho, \varphi}^{\sigma(0), \sigma(1), \ldots}(x)=\sum_{\kappa=0}^{\infty} \frac{\sigma(\kappa)}{\Gamma(\rho \kappa+\varphi)} x^{\kappa}(\rho, \varphi>0 ;|x|<\mathbb{R}),
$$


where $\mathbb{R}$ is the set of real numbers and the coefficients $\sigma(\kappa)\left(\kappa \in \mathbb{N}_{0}=\mathbb{N} \cup\{0\}\right)$ is the bounded sequence of positive real numbers. With the aid of (3), author [28] proposed the following left and right-sided fractional integral operators, respectively, as follows:

$$
\mathcal{J}_{\rho, \varphi, e_{1}^{+} ; \omega}^{\sigma} F(x)=\int_{e_{1}}^{x}(x-\ell)^{\varphi-1} \mathcal{F}_{\rho, \varphi}^{\sigma}\left[\omega(x-\ell)^{\rho}\right] F(\ell) d \ell \quad\left(x>e_{1}\right),
$$

and

$$
\mathcal{J}_{\rho, \varphi, e_{1}^{-} ; \omega}^{\sigma} F(x)=\int_{x}^{e_{2}}(\ell-x)^{\varphi-1} \mathcal{F}_{\rho, \varphi}^{\sigma}\left[\omega(\ell-x)^{\rho}\right] F(\ell) d \ell \quad\left(x<e_{2}\right),
$$

where $\varphi, \rho>0, \omega \in \mathbb{R}$ and $F(\ell)$ is such that the integrals on the right side exists.

The operators mentioned in (4) and (5) are bounded on $L_{1}\left(e_{1}, e_{2}\right)$, if

$$
\bar{m}:=\mathcal{F}_{\rho, \varphi+1}^{\sigma}\left[\mathcal{\omega}(x-\ell)^{\rho}\right]<\infty .
$$

In view of $F \in L_{1}\left(e_{1}, e_{2}\right)$, we have

$$
\left\|\mathcal{J}_{\rho, \varphi, e_{1}^{+} ; \omega}^{\sigma} F\right\|_{1} \leq \bar{m}\left(e_{2}-e_{1}\right)^{\varphi}\|F\|_{1}
$$

and

$$
\left\|\mathcal{J}_{\rho, \varphi, e_{1}^{-} ; \omega}^{\sigma} F\right\|_{1} \leq \bar{m}\left(e_{2}-e_{1}\right)^{\varphi}\|F\|_{1}
$$

where

$$
\|F\|_{\mathcal{P}}:=\left(\int_{e_{1}}^{e_{2}}\left|F(\ell)^{\mathcal{P}} d \ell\right|\right)^{\frac{1}{\mathcal{P}}} .
$$

In fact, the significance of these operators curtails over-simplification. Numerous helpful integral operators can be obtained by specialising in the coefficient $\sigma(\kappa)$.

Here, we just mention that the left and right classical RL-fractional integrals of $\alpha$ th order by replacing the values $\varphi=\alpha, \sigma(0)=1$ and $\omega=0$ in (4) and (5) as follows

$$
\mathcal{I}_{e_{1}^{+}}^{\alpha} F(x)=\int_{e_{1}}^{x}(x-\ell)^{\alpha-1} F(\ell) d \ell \quad\left(x>e_{1}\right)
$$

and

$$
\mathcal{I}_{e_{2}^{-}}^{\alpha} F(x)=\int_{x}^{e_{2}}(\ell-x)^{\alpha-1} F(\ell) d \ell \quad\left(x<e_{2}\right) .
$$

To derive inequality like (2), several researchers established different results pertaining to convexity and fractional integral operators as follows:

Lemma 1 ([29]). Let there be a differentiable mapping $F: \mathbb{I} \subset \mathbb{R} \rightarrow \mathbb{R}$ on $\mathbb{I}^{\circ}$ with $e_{1}<e_{2}$ and $e_{1}, e_{1} \in \mathbb{I}$. If $F^{\prime} \in L_{1}\left(\left[e_{1}, e_{2}\right]\right)$, then the following identity

$$
F(x)-\frac{1}{e_{2}-e_{1}} \int_{e_{1}}^{e_{2}} F(\ell) d \ell=\frac{\left(x-e_{1}\right)^{2}}{e_{2}-e_{1}} \int_{0}^{1} \ell F^{\prime}\left(\ell x+(1-\ell) e_{1}\right) d \ell-\frac{\left(e_{2}-x\right)^{2}}{e_{2}-e_{1}} \int_{0}^{1} \ell F^{\prime}\left(\ell x+(1-\ell) e_{2}\right) d \ell
$$


holds for $x \in\left[e_{1}, e_{2}\right]$.

Lemma 2 ([30]). Let there be a differentiable mapping $F: \mathbb{I} \subset \mathbb{R} \rightarrow \mathbb{R}$ on $\mathbb{I}^{\circ}$ with $e_{1}<e_{2}$ and $e_{1}, e_{1} \in \mathbb{I}$. If $F^{\prime} \in L_{1}\left(\left[e_{1}, e_{2}\right]\right)$, then the following identity

$$
\begin{aligned}
& \frac{\left(x-e_{1}\right)^{\delta}+\left(e_{2}-x\right)^{\delta}}{e_{2}-e_{1}} F(x)-\frac{\Gamma(\delta+1)}{e_{2}-e_{1}}\left[\mathcal{I}_{e_{1}^{-}}^{\delta} F\left(e_{1}\right)+\mathcal{I}_{e_{1}^{+}}^{\delta} F\left(e_{2}\right)\right] \\
& =\frac{\left(x-e_{1}\right)^{\delta+1}}{e_{2}-e_{1}} \int_{0}^{1} \ell^{\delta} F^{\prime}\left(\ell x+(1-\ell) e_{1}\right) d \ell-\frac{\left(e_{2}-x\right)^{\delta+1}}{e_{2}-e_{1}} \int_{0}^{1} \ell^{\alpha} F^{\prime}\left(\ell x+(1-\ell) e_{2}\right) d \ell
\end{aligned}
$$

holds for $x \in\left[e_{1}, e_{2}\right]$.

Lemma 3 ([31]). Let $\wp>0$ and there be a differentiable mapping $F: \mathbb{I} \subset \mathbb{R} \rightarrow \mathbb{R}$ on $\mathbb{I}^{\circ}$ with $e_{1}<e_{2}$ and $e_{1}, e_{1} \in \mathbb{I}$. If $F^{\prime} \in L_{1}\left(\left[e_{1}, e_{2}\right]\right)$, then the following identity

$$
\begin{aligned}
& -\frac{\left(x^{\wp}-e_{1}^{\wp}\right)^{\delta} F\left(e_{1}\right)+\left(e_{2}^{\wp}-x^{\wp}\right)^{\delta} F\left(e_{2}\right)}{e_{2}-e_{1}}-\frac{\Gamma(\delta+1)}{e_{2}-e_{1}}\left[\mathcal{I}_{e_{1}^{\wp}, \delta}^{\wp} F\left(e_{1}\right)+\mathcal{I}_{e_{1}^{+}}^{\wp, \delta} F\left(e_{2}\right)\right] \\
& =\frac{\left(x^{\wp}-e_{1}^{\wp}\right)^{\delta+1}}{\wp^{\delta+1}\left(e_{2}-e_{1}\right)} \int_{0}^{1} \ell^{\delta} \frac{F^{\prime}\left(\wp \sqrt{\ell e_{1}+(1-\ell) x^{\wp}}\right)}{\left(\ell e_{1}^{\wp}+(1-\ell) x^{\wp}\right)^{1-\frac{1}{\wp}}} d \ell-\frac{\left(e_{2}^{\wp}-x^{\wp}\right)^{\delta+1}}{\wp^{1+\delta}\left(e_{2}-e_{1}\right)} \int_{0}^{1} \ell^{\delta} \frac{F^{\prime}\left(\wp \sqrt{\ell e_{2}+(1-\ell) x^{\wp}}\right)}{\left(\ell e_{2}^{\wp}+(1-\ell) x^{\wp}\right)^{1-\frac{1}{\wp}}} d \ell
\end{aligned}
$$

holds for $x \in\left[e_{1}, e_{2}\right]$.

Lemma 4 ([32]). Let $\delta, \wp>0$ and there be a differentiable mapping $F: \mathbb{I} \subset \mathbb{R} \rightarrow \mathbb{R}$ on $\mathbb{I}^{\circ}$ with $e_{1}<e_{2}$ and $e_{1}, e_{1} \in \mathbb{I}$. If $F^{\prime} \in L_{1}\left(\left[e_{1}, e_{2}\right]\right)$, then the following identity

$$
\begin{aligned}
& \frac{\left(x^{\wp}-e_{1}^{\wp}\right)^{\delta} F\left(e_{1}\right)+\left(e_{2}^{\wp}-x^{\wp}\right)^{\delta} F\left(e_{2}\right)}{e_{2}-e_{1}}-\frac{\wp^{\alpha} \Gamma(\delta+1)}{e_{2}-e_{1}}\left[\mathcal{I}_{x^{-}}^{\wp, \delta} F\left(e_{1}\right)+\mathcal{I}_{x^{+}}^{\wp, \delta} F\left(e_{2}\right)\right] \\
& =\frac{\left(x^{\wp}-e_{1}^{\wp}\right)^{\delta+1}}{e_{2}-e_{1}} \int_{0}^{1}\left(\ell^{\delta}-1\right) \frac{F^{\prime}\left(\sqrt{\ell x^{\wp}+(1-\ell) e_{1}^{\wp}}\right)}{\left(\ell x^{\wp}+(1-\ell) e_{1}^{\wp}\right)^{1-\frac{1}{\wp}}} d \ell \\
& \quad+\frac{\left(e_{2}^{\wp}-x^{\wp}\right)^{\delta+1}}{e_{2}-e_{1}} \int_{0}^{1}(1-\ell)^{\delta} \frac{F^{\prime}\left({ }^{\ell x^{\wp}+(1-\ell) b^{\wp}}\right)}{\left(\ell x^{\wp}+(1-\ell) e_{2}^{\wp}\right)^{1-\frac{1}{\wp}}} d \ell
\end{aligned}
$$

holds for $x \in\left[e_{1}, e_{2}\right]$.

It is incontestable that fractional integral inequalities have played a vital role in pure and applied analysis. Recently, the investigation of some well-known integral inequalities for generalised fractional integral has been established by several researchers, (see [31,32]). In [33], Rashid et al. obtained the inequality similar to (2) via $K$-fractional integral operator. Chu et al. [22] derived the novel fractal bounds via generalised exponentially harmonically $s$-convex functions. Thatsatian et al. [31] proved the inequality similar to (2) by employing a generalised fractional integral operator. In [32], Gürbüz et al. established some inequalities by fractional integrals of positive real orders.

Inspired by the above works, here we established the fractional integral inequalities for $\wp$-convex mappings by employing a generalised fractional integral operator depending on the Raina's function. A new integral identity correlated with generalised fractional integral operator is presented. Several estimates of upper bounds concerned with Ostrowski type inequalities are derived. The consequences established here, being very general, are figured out to be specified to produce several existing results for classical convex and harmonically convex mappings. Pertinent relations of the numerous outcomes established here with 
those comprising comparatively simple fractional integral operators are also directed. Moreover, the proposed scheme is supported by applications to apply all established novel outcomes and validate their supremacy.

The following Lemma will be necessary for proving our results:

Lemma 5 ([34]). For $Y_{1} \geq 0, Y_{2} \geq 0$, we have

$$
\text { (i) }\left(Y_{1}+Y_{2}\right)^{a} \leq 2^{a-1}\left(Y_{1}^{a}+Y_{2}^{a}\right), \quad a \geq 1
$$

and

$$
\text { (ii) }\left(Y_{1}+Y_{2}\right)^{a} \leq Y_{1}^{a}+Y_{2}^{a}, \quad 0<a \leq 1 .
$$

\section{Hermite-Hadamard Type Inequality for $\wp$-Convex Functions}

In what follows, our first result is the Hermite-Hadamard type inequality via generalised fractional integral operator for $\wp$-convex functions.

Theorem 1. For $\wp>0$ and let there be a $\wp$-convex mapping $F: \Omega=\left[e_{1}, e_{2}\right] \subseteq(0, \infty) \rightarrow \mathbb{R}$ with $e_{1}, e_{2} \in \Omega$ and $e_{1}<e_{2}$ such that $F \in L_{1}\left(\left[e_{1}, e_{2}\right]\right)$, then the following inequality holds:

$$
\begin{aligned}
F\left(\wp \sqrt{\frac{e_{1}^{\wp}+e_{2}^{\wp}}{2}}\right) & \leq \frac{1}{2\left(e_{2}^{\wp}-e_{1}^{\wp}\right)^{\varphi} \mathcal{F}_{\rho, \varphi+1}^{\sigma}\left(\omega\left(e_{2}^{\wp}-e_{1}^{\wp}\right)^{\rho}\right)}\left\{\mathcal{J}_{\rho, \varphi,\left(e_{1}^{\wp}\right)^{+} ; \omega}^{\sigma} F\left(\sqrt{e_{2}}\right)+\mathcal{J}_{\rho, \varphi,\left(e_{2}^{\wp}\right)-; \omega}^{\sigma} F\left({ }^{\wp} \sqrt{e_{1}}\right)\right\} \\
& \leq \frac{F\left(e_{1}\right)+F\left(e_{2}\right)}{2} .
\end{aligned}
$$

Proof. By the $\wp$-convexity of $F$, we have

$$
\begin{aligned}
F\left(\wp \sqrt{\frac{e_{1}^{\wp}+e_{2}^{\wp}}{2}}\right) & =F\left(\frac{\wp \sqrt{\ell e_{1}^{\wp}+(1-\ell) e_{2}^{\wp}}+\wp \sqrt{(1-\ell) e_{1}^{\wp}+\ell e_{2}^{\wp}}}{2}\right) \\
& \leq \frac{1}{2}\left[F\left(\sqrt{\ell e_{1}^{\wp}+(1-\ell) e_{2}^{\wp}}\right)+F\left(\sqrt{(1-\ell) e_{1}^{\wp}+\ell e_{2}^{\wp}}\right]\right) .
\end{aligned}
$$

Conducting product on both sides by $\ell^{\varphi-1} \mathcal{F}_{\rho, \varphi}^{\sigma}\left(\omega\left(e_{2}^{\wp}-e_{1}^{\wp}\right) \ell^{\rho}\right)$ and then integrating over $[0,1]$, we have

$$
\begin{aligned}
& 2 F\left(\sqrt[\wp]{\frac{e_{1}^{\wp}+e_{2}^{\wp}}{2}}\right) \int_{0}^{1} \ell^{\varphi-1} \mathcal{F}_{\rho, \varphi}^{\sigma}\left(\omega\left(e_{2}^{\wp}-e_{1}^{\wp}\right) \ell^{\rho}\right) d \ell \\
& \leq \int_{0}^{1} \ell^{\varphi-1} \mathcal{F}_{\rho, \varphi}^{\sigma}\left(\omega\left(e_{2}^{\wp}-e_{1}^{\wp}\right) \ell^{\rho}\right)\left[F\left(\wp \sqrt{\ell e_{1}^{\wp}+(1-\ell) e_{2}^{\wp}}\right)+F\left(\sqrt{(1-\ell) e_{1}^{\wp}+\ell e_{2}^{\wp}}\right)\right] d \ell .
\end{aligned}
$$

Changing variable technique, we have 


$$
\begin{aligned}
& 2 F\left(\wp \sqrt{\frac{e_{1}^{\wp}+e_{2}^{\wp}}{2}}\right) \\
& \leq \frac{1}{\left(e_{2}^{\wp}-e_{1}^{\wp}\right)^{\varphi} \mathcal{F}_{\rho, \varphi+1}^{\sigma}\left(\mathscr{\omega}\left(e_{2}^{\wp}-e_{1}^{\wp}\right)^{\rho}\right)}\left\{\int_{e_{1}^{\wp}}^{e_{2}^{\wp}}\left(e_{2}^{\wp}-x\right)^{\varphi-1} \mathcal{F}_{\rho, \varphi}^{\sigma}\left(\omega\left(e_{2}^{\wp}-x^{\wp}\right)\right) F\left(x^{1 / \wp}\right) d x\right. \\
& \left.+\int_{e_{1}^{\wp}}^{e_{2}^{\wp}}\left(x-e_{1}^{\wp}\right)^{\varphi-1} \mathcal{F}_{\rho, \varphi}^{\sigma}\left(\mathfrak{\omega}\left(x^{\wp}-e_{2}^{\wp}\right)\right) F\left(x^{1 / \wp}\right) d x\right\} \\
& =\frac{1}{\left(e_{2}^{\wp}-e_{1}^{\wp}\right)^{\varphi} \mathcal{F}_{\rho, \varphi+1}^{\sigma}\left(\omega\left(e_{2}^{\wp}-e_{1}^{\wp}\right)^{\rho}\right)}\left\{\mathcal{J}_{\rho, \varphi,\left(e_{1}^{\wp}\right)^{+} ; \omega}^{\sigma} F\left(e_{2}^{1 / \wp}\right)+\mathcal{J}_{\rho, \varphi,\left(e_{2}^{\wp}\right)^{-} ; \omega}^{\sigma} F\left(e_{1}^{1 / \wp}\right)\right\} .
\end{aligned}
$$

It follows upon utilising the term-wise integration

$$
\begin{aligned}
\int_{0}^{1} \ell^{\varphi-1} \mathcal{F}_{\rho, \varphi}^{\sigma}\left(\omega\left(e_{2}^{\wp}-e_{1}^{\wp}\right) \ell^{\rho}\right) d \ell & =\sum_{\kappa=0}^{\infty} \frac{\sigma(\kappa) \omega^{\kappa}\left(e_{2}^{\wp}-e_{1}^{\wp}\right)^{\rho \kappa}}{\Gamma(\rho \kappa+\varphi)} \int_{0}^{1} \ell^{\rho \kappa+\varphi-1} d \ell \\
& =\sum_{\kappa=0}^{\infty} \frac{\sigma(\kappa) \omega^{\kappa}\left(e_{2}^{\wp}-e_{1}^{\wp}\right)^{\rho \kappa}}{\Gamma(\rho \kappa+\varphi+1)}=\mathcal{F}_{\rho, \varphi+1}^{\sigma}\left(\omega\left(e_{2}^{\wp}-e_{1}^{\wp}\right)^{\rho}\right) .
\end{aligned}
$$

Since $F$ is $\wp$-convex on $\left[e_{1}, e_{2}\right]$, we have

$$
F\left(\left[\ell e_{1}^{\wp}+(1-\ell) e_{2}^{\wp}\right]^{1 / \wp}\right) \leq \ell F\left(e_{1}\right)+(1-\ell) F\left(e_{2}\right)
$$

and

$$
F\left(\left[(1-\ell) e_{1}^{\wp}+\ell e_{2}^{\wp}\right]^{1 / \wp}\right) \leq(1-\ell) F\left(e_{1}\right)+\ell F\left(e_{2}\right) .
$$

Adding inequalities (20) and (21), multiplying the resulting inequality by $\ell^{\varphi-1} \mathcal{F}_{\rho, \varphi}^{\sigma}$ $\left(\omega\left(e_{2}^{\wp}-e_{1}^{\wp}\right) \ell^{\rho}\right)$ and then integrating over $[0,1]$, we have

$$
\begin{aligned}
& \int_{0}^{1} \ell^{\varphi-1} \mathcal{F}_{\rho, \varphi}^{\sigma}\left(\omega\left(e_{2}^{\wp}-e_{1}^{\wp}\right) \ell^{\rho}\right) F\left(\left[\ell e_{1}^{\wp}+(1-\ell) e_{2}^{\wp}\right]^{1 / \wp}\right) d \ell \\
& \quad+\int_{0}^{1} \ell^{\varphi-1} \mathcal{F}_{\rho, \varphi}^{\sigma}\left(\omega\left(e_{2}^{\wp}-e_{1}^{\wp}\right) \ell^{\rho}\right) F\left(\left[(1-\ell) e_{1}^{\wp}+\ell e_{2}^{\wp}\right]^{1 / \wp}\right) \\
& \leq\left[F\left(e_{1}\right)+F\left(e_{2}\right)\right] \int_{0}^{1} \ell^{\varphi-1} \mathcal{F}_{\rho, \varphi}^{\sigma}\left(\omega\left(e_{2}^{\wp}-e_{1}^{\wp}\right) \ell^{\rho}\right) d \ell .
\end{aligned}
$$

Again, making change of variable, we get

$$
\frac{1}{\left(e_{2}^{\wp}-e_{1}^{\wp}\right)^{\varphi} \mathcal{F}_{\rho, \varphi+1}^{\sigma}\left(\omega\left(e_{2}^{\wp}-e_{1}^{\wp}\right)^{\rho}\right)}\left\{\mathcal{J}_{\rho, \varphi,\left(e_{1}^{\wp}\right)^{+} ; \omega}^{\sigma} F\left(e_{2}^{1 / \wp}\right)+\mathcal{J}_{\rho, \varphi,\left(e_{2}^{\wp}\right)^{-} ; \omega}^{\sigma} F\left(e_{1}^{1 / \wp}\right)\right\} \leq\left[F\left(e_{1}\right)+F\left(e_{2}\right)\right] .
$$

After appropriate arrangements, we get the desired inequality (15). 
Remark 1. Theorem 1 leads to the conclusions that:

I. letting $\wp=1=\sigma(0), \varphi=\delta$ and $\omega=0$, then we have a little simpler inequality (1).

II. letting $\wp=-1, \sigma(0)=1, \varphi=\delta$ and $\omega=0$, then we have a little simpler inequality obtained by [35].

\section{Ostrowski Type Inequalitiy}

Firstly, we present a lemma for differentiable mappings which is a basic tool to obtain our main consequences. Then, we will show certain estimates which are the modifications of earlier works.

To prove our main consequences, we need the following lemma.

Lemma 6. For $\alpha \in[0,1]$ and $\wp>0$ and let there be a differentiable function $F: \Omega \subseteq(0, \infty) \rightarrow \mathbb{R}$ on $\Omega^{\circ}$ (the interior of $\Omega$ ) with $e_{1}<e_{2}$. If $F \in L_{1}\left(\left[e_{1}, e_{2}\right]\right)$. Then the following identity holds:

$$
\begin{aligned}
& (1-\alpha) \wp \frac{\left(x^{\wp}-e_{1}^{\wp}\right)^{\varphi}+\left(e_{2}^{\wp}-x^{\wp}\right)^{\varphi}}{\mathcal{F}_{\rho, \varphi+1}^{\sigma}\left[\omega\left(e_{2}^{\wp}-e_{1}^{\wp}\right)^{\rho}\right]} F(x)+\alpha \wp \frac{\left(x^{\wp}-e_{1}^{\wp}\right)^{\varphi} F\left(e_{1}\right)+\left(e_{2}^{\wp}-x^{\wp}\right)^{\varphi} F\left(e_{2}\right)}{\mathcal{F}_{\rho, \varphi+1}^{\sigma}\left[\omega\left(e_{2}^{\wp}-e_{1}^{\wp}\right)^{\rho}\right]} \\
& -\frac{\wp^{\varphi+1}}{\mathcal{F}_{\rho, \varphi+1}^{\sigma}\left[\mathcal{\omega}\left(e_{2}^{\wp}-e_{1}^{\wp}\right)^{\rho}\right]}\left[\mathcal{J}_{\rho, \varphi, x^{-} ; \omega}^{\sigma} F\left(e_{1}\right)+\mathcal{J}_{\rho, \varphi, x^{+} ; \omega^{\sigma}}^{\sigma} F\left(e_{2}\right)\right] \\
& =\frac{\left(x^{\wp}-e_{1}^{\wp}\right)^{\varphi+1}}{\mathcal{F}_{\rho, \varphi+1}^{\sigma}\left[\omega\left(e_{2}^{\wp}-e_{1}^{\wp}\right)^{\rho}\right]} \int_{0}^{1}\left(\ell^{\varphi}-\alpha\right) \mathcal{F}_{\rho, \varphi+1}^{\sigma}\left[\omega\left(e_{2}^{\wp}-e_{1}^{\wp}\right)^{\rho}\left(\ell^{\rho}-\alpha\right)\right]\left(\ell x^{\wp}+(1-\ell) e_{1}^{\wp}\right)^{\frac{1}{\wp}-1} F^{\prime}\left(\sqrt{\ell x^{\wp}+(1-\ell) e_{1}^{\wp}}\right) d \ell \\
& -\frac{\left(e_{2}^{\wp}-x^{\wp}\right)^{\varphi+1}}{\mathcal{F}_{\rho, \varphi+1}^{\sigma}\left[\omega\left(e_{2}^{\wp}-e_{1}^{\wp}\right)^{\rho}\right]} \int_{0}^{1}\left(\ell^{\varphi}-\alpha\right) \mathcal{F}_{\rho, \varphi+1}^{\sigma}\left[\omega\left(e_{2}^{\wp}-e_{1}^{\wp}\right)^{\rho}\left(\ell^{\rho}-\alpha\right)\right]\left(\ell x^{\wp}+(1-\ell) e_{2}^{\wp}\right)^{\frac{1}{\zeta}-1} F^{\prime}\left(\wp \sqrt{\ell x^{\wp}+(1-\ell) e_{2}^{\wp}}\right) d \ell .
\end{aligned}
$$

Proof. Integrating by parts, we have

$$
\begin{aligned}
& \int_{0}^{1}\left(\ell^{\varphi}-\alpha\right) \mathcal{F}_{\rho, \varphi+1}^{\sigma}\left[\omega\left(e_{2}^{\wp}-e_{1}^{\wp}\right)^{\rho}\left(\ell^{\rho}-\alpha\right)\right]\left(\ell x^{\wp}+(1-\ell) e_{1}^{\wp}\right)^{\frac{1}{\wp}-1} F^{\prime}\left(\wp \sqrt{\ell x^{\wp}+(1-\ell) e_{1}^{\wp}}\right) d \ell \\
& =\int_{0}^{1}\left(\ell^{\varphi}-\alpha\right) \mathcal{F}_{\rho, \varphi+1}^{\sigma}\left[\omega\left(e_{2}^{\wp}-e_{1}^{\wp}\right)^{\rho}\left(\ell^{\rho}-\alpha\right)\right]\left(\ell x^{\wp}+(1-\ell) e_{1}^{\wp}\right)^{\frac{1}{\wp}-1} F^{\prime}\left(\wp \sqrt{\ell x^{\wp}+(1-\ell) e_{1}^{\wp}}\right) d \ell \\
& =\left.\wp\left(\ell^{\varphi}-\alpha\right) \mathcal{F}_{\rho, \varphi+1}^{\sigma}\left[\omega\left(e_{2}^{\wp}-e_{1}^{\wp}\right)^{\rho}\left(\ell^{\rho}-\alpha\right)\right] \frac{F\left(\wp \sqrt{\ell x^{\wp}+(1-\ell) e_{1}^{\wp}}\right)}{x^{\wp}-e_{1}^{\wp}}\right|_{0} ^{1} \\
& -\frac{\wp}{x^{\wp}-e_{1}^{\wp}} \int_{0}^{1} \ell^{\varphi-1} \mathcal{F}_{\rho, \varphi}^{\sigma}\left[\omega\left(e_{2}^{\wp}-e_{1}^{\wp}\right)^{\rho} \ell^{\rho}\right] F\left(\sqrt{\ell x^{\wp}+(1-\ell) e_{1}^{\wp}}\right) d \ell \\
& =\frac{\wp \mathcal{F}_{\rho, \varphi+1}^{\sigma}\left[\omega\left(e_{2}^{\wp}-e_{1}^{\wp}\right)^{\rho}\right]}{x^{\wp}-e_{1}^{\wp}}\left[(1-\alpha) F(x)+\alpha F\left(e_{1}\right)\right]-\left(\frac{\wp}{x^{\wp}-e_{1}^{\wp}}\right)^{\varphi+1} \mathcal{J}_{\rho, \varphi, x^{-} ; \omega}^{\sigma} F\left(e_{1}\right) .
\end{aligned}
$$

In a similar way, we have

$$
\begin{aligned}
& \int_{0}^{1}\left(\ell^{\varphi}-\alpha\right) \mathcal{F}_{\rho, \varphi+1}^{\sigma}\left[\omega\left(e_{2}^{\wp}-e_{1}^{\wp}\right)^{\rho}\left(\ell^{\rho}-\alpha\right)\right]\left(\ell x^{\wp}+(1-\ell) e_{2}^{\wp}\right)^{\frac{1}{\wp}-1} F^{\prime}\left(\sqrt{\ell x^{\wp}+(1-\ell) e_{2}^{\wp}}\right) d \ell \\
& =-\frac{\wp \mathcal{F}_{\rho, \varphi+1}^{\sigma}\left[\omega\left(e_{2}^{\wp}-e_{1}^{\wp}\right)^{\rho}\right]}{e_{2}^{\wp}-x^{\wp}}\left[(1-\alpha) F(x)+\alpha F\left(e_{2}\right)\right]-\left(\frac{\wp}{e_{2}^{\wp}-x^{\wp}}\right)^{\varphi+1} \mathcal{J}_{\rho, \varphi, x^{+} ; \omega}^{\sigma} F\left(e_{2}\right) .
\end{aligned}
$$


Multiplying both sides of (24) and (25) by $\frac{\left(x^{\wp}-e_{1}^{\wp}\right)^{\varphi+1}}{\mathcal{F}_{\rho, \varphi+1}^{\sigma}\left[\omega\left(e_{2}^{\wp}-e_{1}^{\wp}\right)^{\rho}\right]}$ and $\frac{\left(e_{2}^{\wp}-x^{\wp}\right)^{\varphi+1}}{\mathcal{F}_{\rho, \varphi+1}^{\sigma}\left[\omega\left(e_{2}^{\wp}-e_{1}^{\wp}\right)^{\rho}\right]}$, respectively, we have

$$
\begin{aligned}
& \frac{\left(x^{\wp}-e_{1}^{\wp}\right)^{\varphi+1}}{\mathcal{F}_{\rho, \varphi+1}^{\sigma}\left[\omega\left(e_{2}^{\wp}-e_{1}^{\wp}\right)^{\rho}\right]} \int_{0}^{1}\left(\ell^{\varphi}-\alpha\right) \mathcal{F}_{\rho, \varphi+1}^{\sigma}\left[\omega\left(e_{2}^{\wp}-e_{1}^{\wp}\right)^{\rho}\left(\ell^{\rho}-\alpha\right)\right]\left(\ell x^{\wp}+(1-\ell) e_{1}^{\wp}\right)^{\frac{1}{\wp}-1} F^{\prime}\left(\sqrt{\ell x^{\wp}+(1-\ell) e_{1}^{\wp}}\right) d \ell \\
& =\frac{\wp\left(x^{\wp}-e_{1}^{\wp}\right)^{\varphi}}{\mathcal{F}_{\rho, \varphi+1}^{\sigma}\left[\omega\left(e_{2}^{\wp}-e_{1}^{\wp}\right)^{\rho}\right]}(1-\alpha) F(x)+\frac{\wp\left(x^{\wp}-e_{1}^{\wp}\right)^{\varphi}}{\mathcal{F}_{\rho, \varphi+1}^{\sigma}\left[\omega\left(e_{2}^{\wp}-e_{1}^{\wp}\right)^{\rho}\right]} \alpha F\left(e_{1}\right)-\frac{\wp^{\varphi+1}}{\mathcal{F}_{\rho, \varphi+1}^{\sigma}\left[\omega\left(e_{2}^{\wp}-e_{1}^{\wp}\right)^{\rho}\right]} \mathcal{J}_{\rho, \varphi, x^{-} ; \omega}^{\sigma} F\left(e_{1}\right) .
\end{aligned}
$$

and

$$
\begin{aligned}
& \frac{\left(e_{2}^{\wp}-x^{\wp}\right)^{\varphi+1}}{\mathcal{F}_{\rho, \varphi+1}^{\sigma}\left[\omega\left(e_{2}^{\wp}-e_{1}^{\wp}\right)^{\rho}\right]} \int_{0}^{1}\left(\ell^{\varphi}-\alpha\right) \mathcal{F}_{\rho, \varphi+1}^{\sigma}\left[\omega\left(e_{2}^{\wp}-e_{1}^{\wp}\right)^{\rho}\left(\ell^{\rho}-\alpha\right)\right]\left(\ell x^{\wp}+(1-\ell) e_{2}^{\wp}\right)^{\frac{1}{\wp}-1} F^{\prime}\left(\sqrt{\ell x}+(1-\ell) e_{2}^{\wp}\right) d \ell \\
= & -\frac{\wp\left(e_{2}^{\wp}-x^{\wp}\right)^{\varphi}}{\mathcal{F}_{\rho, \varphi+1}^{\sigma}\left[\omega\left(e_{2}^{\wp}-e_{1}^{\wp}\right)^{\rho}\right]}(1-\alpha) F(x)-\frac{\wp\left(e_{2}^{\wp}-x^{\wp}\right)^{\varphi}}{\mathcal{F}_{\rho, \varphi+1}^{\sigma}\left[\omega\left(e_{2}^{\wp}-e_{1}^{\wp}\right)^{\rho}\right]} \alpha F\left(e_{1}\right)-\frac{\wp^{\varphi+1}}{\mathcal{F}_{\rho, \varphi+1}^{\sigma}\left[\omega\left(e_{2}^{\wp}-e_{1}^{\wp}\right)^{\rho}\right]} \mathcal{J}_{\rho, \varphi, x^{+} ; \omega}^{\sigma} F\left(e_{1}\right) . \quad(27)
\end{aligned}
$$

Adding (26) and (27) gives the desired equality. So, this completes the proof.

Remark 2. Lemma 6 leads to the following conclusions that:

I. letting $\alpha=1, \varphi=\delta, \sigma(0)=1$ and $\omega=0$, then we get Lemma 4 .

II. letting $\wp=1, \varphi=\delta, \sigma(0)=1$ and $\alpha=\omega=0$, then we get Lemma 1 .

III. letting $=\wp=\delta=1, \varphi=\delta, \sigma(0)=1$ and $\wp=\omega=0$, then we get Lemma 2 .

Throughout this investigation, for the sake of simplicity, we denote

$$
\begin{aligned}
\mathrm{Y}_{F}\left(\wp ; \alpha, \varphi, \rho, \sigma ; e_{1}, e_{2}\right)=\quad(1 & -\alpha) \wp \frac{\left(x^{\wp}-e_{1}^{\wp}\right)^{\varphi}+\left(e_{2}^{\wp}-x^{\wp}\right)^{\varphi}}{\mathcal{F}_{\rho, \varphi+1}^{\sigma}\left[\omega\left(e_{2}^{\wp}-e_{1}^{\wp}\right)^{\rho}\right]} F(x)+\alpha \wp \frac{\left(x^{\wp}-e_{1}^{\wp}\right)^{\varphi} F\left(e_{1}\right)+\left(e_{2}^{\wp}-x^{\wp}\right)^{\varphi} F\left(e_{2}\right)}{\mathcal{F}_{\rho, \varphi+1}^{\sigma}\left[\omega\left(e_{2}^{\wp}-e_{1}^{\wp}\right)^{\rho}\right]} \\
& -\frac{\wp^{\varphi+1}}{\mathcal{F}_{\rho, \varphi+1}^{\sigma}\left[\omega\left(e_{2}^{\wp}-e_{1}^{\wp}\right)^{\rho}\right]}\left[\mathcal{J}_{\rho, \varphi, x^{-} ; \omega}^{\sigma} F\left(e_{1}\right)+\mathcal{J}_{\rho, \varphi, x^{+} ; \omega}^{\sigma} F\left(e_{2}\right)\right],
\end{aligned}
$$

unless otherwise specified.

The incomplete beta function:

$$
\mathbb{B}_{a}(X, Y)=\int_{0}^{a} \ell^{X-1}(1-\ell)^{Y-1} d \ell, X, Y>0, a \in(0,1)
$$

The following computations of definite integrals are required in Theorem 2. 


$$
\begin{aligned}
& \Theta_{1}(\beta) \quad:=\int_{0}^{1} \ell\left|\ell^{\varphi}-\alpha\right| \mathcal{F}_{\rho, \varphi+1}^{\sigma}\left[\omega\left(e_{2}^{\wp}-e_{1}^{\wp}\right)^{\rho}\left(\ell^{\rho}-\alpha\right)\right]\left(\ell^{\frac{1}{\varsigma}-1} x^{1-\wp}+(1-\ell)^{\frac{1}{\wp}-1} \beta^{1-\wp}\right) d \ell \\
& =\int_{0}^{\alpha^{1 / \varphi}} \ell\left(\alpha-\ell^{\varphi}\right) \mathcal{F}_{\rho, \varphi+1}^{\sigma}\left[\omega\left(e_{2}^{\wp}-e_{1}^{\wp}\right)^{\rho}\left(\ell^{\rho}-\alpha\right)\right]\left(\ell^{\frac{1}{\zeta}-1} x^{1-\wp}+(1-\ell)^{\frac{1}{\varphi}-1} \beta^{1-\wp}\right) d \ell \\
& +\int_{\alpha^{1 / \varphi}}^{1} \ell\left(\ell^{\varphi}-\alpha\right) \mathcal{F}_{\rho, \varphi+1}^{\sigma}\left[\omega\left(e_{2}^{\wp}-e_{1}^{\wp}\right)^{\rho}\left(\ell^{\rho}-\alpha\right)\right]\left(\ell^{\frac{1}{\varsigma}-1} x^{1-\wp}+(1-\ell)^{\frac{1}{\wp}-1} \beta^{1-\wp}\right) d \ell \\
& =\sum_{\kappa=0}^{\infty} \frac{\sigma(\kappa) \omega^{\kappa}\left(e_{2}^{\wp}-e_{1}^{\wp}\right)}{\Gamma(\rho \kappa+\varphi+1)}\left\{\int_{0}^{\alpha^{1 / \varphi}}\left(\alpha \ell^{1+\rho \kappa}-\ell^{\kappa \rho+\varphi+1}\right)\left(\ell^{\frac{1}{\wp}-1} x^{1-\wp}+(1-\ell)^{\frac{1}{\wp}-1} \beta^{1-\wp}\right) d \ell\right. \\
& \left.+\int_{\alpha^{1 / \varphi}}^{1}\left(\ell^{\kappa \rho+\varphi+1}-\alpha \ell^{1+\rho \kappa}\right)\left(\ell^{\frac{1}{\varsigma}-1} x^{1-\wp}+(1-\ell)^{\frac{1}{\wp}-1} \beta^{1-\wp}\right) d \ell\right\} \\
& =\sum_{\kappa=0}^{\infty} \frac{\sigma(\kappa) \omega^{\kappa}\left(e_{2}^{\wp}-e_{1}^{\wp}\right)}{\Gamma(\rho \kappa+\varphi+1)}\left\{\frac{x^{1-\wp} \alpha^{\rho \kappa \wp+\varphi+\wp \varphi}}{\rho \kappa+\frac{1}{\wp}+1}+\alpha \beta^{\wp-1} \mathbb{B}_{\alpha^{1 / \varphi}}(\rho \kappa+2,1 / \wp)-\beta^{\wp-1} \mathbb{B}_{\alpha^{1 / \varphi}}(\kappa \rho+\varphi+2,1 / \wp)\right. \\
& \left.+\beta^{\wp-1} \mathbb{B}_{1-\alpha^{1 / \varphi}}(1 / \wp, \rho \kappa+\varphi+2)+\frac{\alpha x^{1-\wp} \alpha^{\frac{\rho \kappa \wp+\varphi+\wp \varphi}{\wp \varphi}}}{\rho \kappa+\frac{1}{\wp}+1}-\alpha \beta^{\wp-1} \mathbb{B}_{1-\alpha^{1 / \varphi}}(1 / \wp, \rho \kappa+2)\right\}
\end{aligned}
$$

and

$$
\begin{aligned}
\Theta_{2}(\beta) \quad:= & \int_{0}^{1}\left|\ell^{\varphi}-\alpha\right| \mathcal{F}_{\rho, \varphi+1}^{\sigma}\left[\omega\left(e_{2}^{\wp}-e_{1}^{\wp}\right)^{\rho}\left(\ell^{\rho}-\alpha\right)\right]\left(\ell^{\frac{1}{\wp}-1} x^{1-\wp}+(1-\ell)^{\frac{1}{\wp}-1} \beta^{1-\wp}\right) d \ell \\
= & \int_{0}^{\alpha^{1 / \varphi}}\left|\alpha-\ell^{\varphi}\right| \mathcal{F}_{\rho, \varphi+1}^{\sigma}\left[\omega\left(e_{2}^{\wp}-e_{1}^{\wp}\right)^{\rho}\left(\ell^{\rho}-\alpha\right)\right]\left(\ell^{\frac{1}{\varphi}-1} x^{1-\wp}+(1-\ell)^{\frac{1}{\wp}-1} \beta^{1-\wp}\right) d \ell \\
& +\int_{\alpha^{1 / \varphi}}^{1}\left|\ell^{\varphi}-\alpha\right| \mathcal{F}_{\rho, \varphi+1}^{\sigma}\left[\omega\left(e_{2}^{\wp}-e_{1}^{\wp}\right)^{\rho}\left(\ell^{\rho}-\alpha\right)\right]\left(\ell^{\frac{1}{\zeta}-1} x^{1-\wp}+(1-\ell)^{\frac{1}{\wp}-1} \beta^{1-\wp}\right) d \ell \\
= & \sum_{\kappa=0}^{\infty} \frac{\sigma(\kappa) \omega^{\kappa}\left(e_{2}^{\wp}-e_{1}^{\wp}\right)}{\Gamma(\rho \kappa+\varphi+1)}\left\{\frac{\alpha x^{1-\wp} \alpha^{\frac{2 \rho \kappa \rho+\varphi}{\varphi \varphi}}}{\rho \kappa+\frac{1}{\wp}}+\alpha \beta^{1-\wp} \mathbb{B}_{\alpha^{1 / \varphi}}(\rho \kappa+1,1 / \wp)-\frac{2 x^{1-\wp} \alpha^{\rho \kappa \wp+\varphi+\wp}}{\varphi+\rho \kappa+\frac{1}{\wp}}\right. \\
& \left.+\beta^{1-\wp} \mathbb{B}_{\alpha^{1 / \varphi}}(\varphi+\rho \kappa+1,1 / \wp)+\beta^{1-\wp} \mathbb{B}_{1-\alpha^{1 / \varphi}}(\varphi+\rho \kappa+1,1 / \wp)-\beta^{1-\wp} \alpha \mathbb{B}_{1-\alpha^{1 / \varphi}}(\rho \kappa+1,1 / \wp)\right\} .
\end{aligned}
$$

Theorem 2. For $0<\wp \leq 1,\left(\frac{1}{2}\right)^{\varphi}<\alpha \leq 1$ and let there be a differentiable function $F: \Omega \subset$ $(0, \infty) \rightarrow \mathbb{R}$ on $\Omega^{\circ}$ with $e_{1}<e_{2}$ such that $F^{\prime} \in L_{1}\left(\left[e_{1}, e_{2}\right]\right)$. If $\left|F^{\prime}\right|$ is $\wp$-convex on $\Omega$, then for all $x \in\left(e_{1}, e_{2}\right)$, the following inequality holds:

(a) For $\wp \in\left(0, \frac{1}{2}\right]$, we have

$$
\begin{aligned}
& \left|\mathrm{Y}_{F}\left(\wp ; \alpha, \varphi, \rho, \sigma ; e_{1}, e_{2}\right)\right| \\
& \leq 2^{\frac{1}{\wp}-2}\left\{\frac{\left(x^{\wp}-e_{1}^{\wp}\right)^{\varphi+1}}{\mathcal{F}_{\rho, \varphi+1}^{\sigma}\left[\mathcal{\omega}\left(e_{2}^{\wp}-e_{1}^{\wp}\right)^{\rho}\right]}\left[\Theta_{1}\left(e_{1}\right)\left|F^{\prime}(x)\right|+\left(\Theta_{2}\left(e_{1}\right)-\Theta_{1}\left(e_{1}\right)\right)\left|F^{\prime}\left(e_{1}\right)\right|\right]\right. \\
& \left.\quad+\frac{\left(e_{2}^{\wp}-x^{\wp}\right)^{\varphi+1}}{\mathcal{F}_{\rho, \varphi+1}^{\sigma}\left[\omega\left(e_{2}^{\wp}-e_{1}^{\wp}\right)^{\rho}\right]}\left[\Theta_{1}\left(e_{2}\right)\left|F^{\prime}(x)\right|+\left(\Theta_{2}\left(e_{2}\right)-\Theta_{1}\left(e_{2}\right)\right)\left|F^{\prime}\left(e_{2}\right)\right|\right]\right\} .
\end{aligned}
$$


(b) For $\wp \in\left(\frac{1}{2}, 1\right]$, we have

$$
\begin{aligned}
& \left|\mathrm{Y}_{F}\left(\wp ; \alpha, \varphi, \rho, \sigma ; e_{1}, e_{2}\right)\right| \\
& \leq \frac{\left(x^{\wp}-e_{1}^{\wp}\right)^{\varphi+1}}{\mathcal{F}_{\rho, \varphi+1}^{\sigma}\left[\omega\left(e_{2}^{\wp}-e_{1}^{\wp}\right)^{\rho}\right]}\left[\Theta_{1}\left(e_{1}\right)\left|F^{\prime}(x)\right|+\left(\Theta_{2}\left(e_{1}\right)-\Theta_{1}\left(e_{1}\right)\right)\left|F^{\prime}\left(e_{1}\right)\right|\right] \\
& \quad+\frac{\left(e_{2}^{\wp}-x^{\wp}\right)^{\varphi+1}}{\mathcal{F}_{\rho, \varphi+1}^{\sigma}\left[\omega\left(e_{2}^{\wp}-e_{1}^{\wp}\right)^{\rho}\right]}\left[\Theta_{1}\left(e_{2}\right)\left|F^{\prime}(x)\right|+\left(\Theta_{2}\left(e_{2}\right)-\Theta_{1}\left(e_{2}\right)\right)\left|F^{\prime}\left(e_{2}\right)\right|\right],
\end{aligned}
$$

where $\Theta_{1}(\beta)$ and $\Theta_{2}(\beta)$ are defined by (29) and (30), respectively.

Proof. (a) For $\wp \in\left(0, \frac{1}{2}\right]$, using Lemma 6 and by $\wp$-convexity of $\left|F^{\prime}\right|$, we have that

$$
\begin{aligned}
& \left|\mathrm{Y}_{F}\left(\wp ; \alpha, \varphi, \rho, \sigma ; e_{1}, e_{2}\right)\right| \\
& \leq \frac{\left(x^{\wp}-e_{1}^{\wp}\right)^{\varphi+1}}{\mathcal{F}_{\rho, \varphi+1}^{\sigma}\left[\omega\left(e_{2}^{\wp}-e_{1}^{\wp}\right)^{\rho}\right]} \int_{0}^{1}\left|\ell^{\varphi}-\alpha\right| \mathcal{F}_{\rho, \varphi+1}^{\sigma}\left[\omega\left(e_{2}^{\wp}-e_{1}^{\wp}\right)^{\rho}\left(\ell^{\rho}-\alpha\right)\right]\left(\ell x^{\wp}+(1-\ell) e_{1}^{\wp}\right)^{\frac{1}{\wp}-1}\left|F^{\prime}\left(\wp \sqrt{\ell x^{\wp}+(1-\ell) e_{1}^{\wp}}\right)\right| d \ell \\
& -\frac{\left(e_{2}^{\wp}-x^{\wp}\right)^{\varphi+1}}{\mathcal{F}_{\rho, \varphi+1}^{\sigma}\left[\omega\left(e_{2}^{\wp}-e_{1}^{\wp}\right)^{\rho}\right]} \int_{0}^{1}\left|\ell^{\varphi}-\alpha\right| \mathcal{F}_{\rho, \varphi+1}^{\sigma}\left[\omega\left(e_{2}^{\wp}-e_{1}^{\wp}\right)^{\rho}\left(\ell^{\rho}-\alpha\right)\right]\left(\ell x^{\wp}+(1-\ell) e_{2}^{\wp}\right)^{\frac{1}{\wp}-1}\left|F^{\prime}\left(\wp \sqrt{\ell x^{\wp}+(1-\ell) e_{2}^{\wp}}\right)\right| d \ell \\
& \leq \frac{\left(x^{\wp}-e_{1}^{\wp}\right)^{\varphi+1}}{\mathcal{F}_{\rho, \varphi+1}^{\sigma}\left[\mathfrak{\omega}\left(e_{2}^{\wp}-e_{1}^{\wp}\right)^{\rho}\right]} \\
& \times\left\{\int_{0}^{1}\left|\ell^{\varphi}-\alpha\right| \mathcal{F}_{\rho, \varphi+1}^{\sigma}\left[\omega\left(e_{2}^{\wp}-e_{1}^{\wp}\right)^{\rho}\left(\ell^{\rho}-\alpha\right)\right]\left(\ell x^{\wp}+(1-\ell) e_{1}^{\wp}\right)^{\frac{1}{\zeta}-1}\left\{\ell\left|F^{\prime}(x)\right|+(1-\ell)\left|F^{\prime}\left(e_{1}\right)\right|\right\} d \ell\right\} \\
& -\frac{\left(e_{2}^{\wp}-x^{\wp}\right)^{\varphi+1}}{\mathcal{F}_{\rho, \varphi+1}^{\sigma}\left[\omega\left(e_{2}^{\wp}-e_{1}^{\wp}\right)^{\rho}\right]} \\
& \times\left\{\int_{0}^{1}\left|\ell^{\varphi}-\alpha\right| \mathcal{F}_{\rho, \varphi+1}^{\sigma}\left[\omega\left(e_{2}^{\wp}-e_{1}^{\wp}\right)^{\rho}\left(\ell^{\rho}-\alpha\right)\right]\left(\ell x^{\wp}+(1-\ell) e_{2}^{\wp}\right)^{\frac{1}{\wp}-1}\left\{\ell\left|F^{\prime}(x)\right|+(1-\ell)\left|F^{\prime}\left(e_{2}\right)\right|\right\} d \ell\right\} .
\end{aligned}
$$

Since $\wp \in\left(0, \frac{1}{2}\right]$, utilising Lemma 5 , we have that

$$
\left(\ell x^{\wp}+(1-\ell) \beta^{\wp}\right)^{\frac{1}{\wp}-1} \leq 2^{\frac{1}{\wp}-2}\left(\ell^{\frac{1}{\wp}-1} x^{1-\wp}+(1-\ell)^{\frac{1}{\wp}-1} \beta^{1-\wp}\right), \quad \forall \ell \in[0,1], \beta \in\left\{e_{1}, e_{2}\right\} .
$$

Therefore, we have

$$
\begin{aligned}
& \int_{0}^{1} \ell\left|\ell^{\varphi}-\alpha\right| \mathcal{F}_{\rho, \varphi+1}^{\sigma}\left[\omega\left(e_{2}^{\wp}-e_{1}^{\wp}\right)^{\rho}\left(\ell^{\rho}-\alpha\right)\right]\left(\ell x^{\wp}+(1-\ell) e_{1}^{\wp}\right)^{\frac{1}{\wp}-1} d \ell \\
& \leq 2^{\frac{1}{\wp}-2} \int_{0}^{1} \ell\left|\ell^{\varphi}-\alpha\right| \mathcal{F}_{\rho, \varphi+1}^{\sigma}\left[\omega\left(e_{2}^{\wp}-e_{1}^{\wp}\right)^{\rho}\left(\ell^{\rho}-\alpha\right)\right]\left(\ell^{\frac{1}{\varsigma}-1} x^{1-\wp}+(1-\ell)^{\frac{1}{\wp}-1} \beta^{1-\wp}\right) d \ell \\
& =2^{\frac{1}{\wp}-2} \Theta_{1}(\beta) \\
& \quad \text { and }
\end{aligned}
$$




$$
\begin{aligned}
& \int_{0}^{1}(1-\ell)\left|\ell^{\varphi}-\alpha\right| \mathcal{F}_{\rho, \varphi+1}^{\sigma}\left[\mathcal{\omega}\left(e_{2}^{\wp}-e_{1}^{\wp}\right)^{\rho}\left(\ell^{\rho}-\alpha\right)\right]\left(\ell x^{\wp}+(1-\ell) e_{1}^{\wp}\right)^{\frac{1}{\zeta}-1} d \ell \\
& \leq 2^{\frac{1}{\wp}-2} \int_{0}^{1}(1-\ell)\left|\ell^{\varphi}-\alpha\right| \mathcal{F}_{\rho, \varphi+1}^{\sigma}\left[\mathcal{\omega}\left(e_{2}^{\wp}-e_{1}^{\wp}\right)^{\rho}\left(\ell^{\rho}-\alpha\right)\right]\left(\ell^{\frac{1}{\wp}-1} x^{1-\wp}+(1-\ell)^{\frac{1}{\wp}-1} \beta^{1-\wp}\right) d \ell \\
& =2^{\frac{1}{\wp}-2}\left(\Theta_{2}(\beta)-\Theta_{1}(\beta)\right) .
\end{aligned}
$$

Combining (34), (35) and (36), we get the desired inequality (31).

To prove $(b)$, let $\wp \in\left(\frac{1}{2}, 1\right]$, then we get the required inequality in (32) by employing the inequality (14)

$$
\left(\ell x^{\wp}+(1-\ell) \beta^{\wp}\right)^{\frac{1}{\wp}-1} \leq\left(\ell^{\frac{1}{\wp}-1} x^{1-\wp}+(1-\ell)^{\frac{1}{\wp}-1} \beta^{1-\wp}\right), \quad \forall \ell \in[0,1], \beta \in\left\{e_{1}, e_{2}\right\} .
$$

Corollary 1. Theorem 2 with $\left|F^{\prime}\right| \leq \mathcal{M}$ reduces to

(a) For $\wp \in\left(0, \frac{1}{2}\right]$, the following inequality holds:

$$
\begin{aligned}
& \left|\mathrm{Y}_{F}\left(\wp ; \alpha, \varphi, \rho, \sigma ; e_{1}, e_{2}\right)\right| \\
& \leq 2^{\frac{1}{\wp}-2} \mathcal{M}\left\{\frac{\left(x^{\wp}-e_{1}^{\wp}\right)^{\varphi+1}}{\mathcal{F}_{\rho, \varphi+1}^{\sigma}\left[\omega\left(e_{2}^{\wp}-e_{1}^{\wp}\right)^{\rho}\right]} \Theta_{2}\left(e_{1}\right)+\frac{\left(e_{2}^{\wp}-x^{\wp}\right)^{\varphi+1}}{\mathcal{F}_{\rho, \varphi+1}^{\sigma}\left[\omega\left(e_{2}^{\wp}-e_{1}^{\wp}\right)^{\rho}\right]} \Theta_{2}\left(e_{2}\right)\right\} .
\end{aligned}
$$

(b) For $\wp \in\left(\frac{1}{2}, 1\right]$, the following inequality holds:

$$
\begin{aligned}
& \left|\mathrm{Y}_{F}\left(\wp ; \alpha, \varphi, \rho, \sigma ; e_{1}, e_{2}\right)\right| \\
& \leq \mathcal{M}\left\{\frac{\left(x^{\wp}-e_{1}^{\wp}\right)^{\varphi+1}}{\mathcal{F}_{\rho, \varphi+1}^{\sigma}\left[\omega\left(e_{2}^{\wp}-e_{1}^{\wp}\right)^{\rho}\right]} \Theta_{2}\left(e_{1}\right)+\frac{\left(e_{2}^{\wp}-x^{\wp}\right)^{\varphi+1}}{\mathcal{F}_{\rho, \varphi+1}^{\sigma}\left[\omega\left(e_{2}^{\wp}-e_{1}^{\wp}\right)^{\rho}\right]} \Theta_{2}\left(e_{2}\right)\right\} .
\end{aligned}
$$

Theorem 3. For $r>1,0<\wp \leq 1,\left(\frac{1}{2}\right)^{\varphi}<\alpha \leq 1$ and let there be a differentiable function $F: \Omega \subset(0, \infty) \rightarrow \mathbb{R}$ on $\Omega^{\circ}$ with $e_{1}<e_{2}$ such that $F^{\prime} \in L_{1}\left(\left[e_{1}, e_{2}\right]\right)$. If $\left|F^{\prime}\right|^{r}$ is $\wp$-convex on $\Omega$, then for all $x \in\left(e_{1}, e_{2}\right)$, the following inequality holds:

(a) For $\wp \in\left(0, \frac{1}{2}\right]$, we have

$$
\begin{aligned}
& \left|Y_{F}\left(\wp ; \alpha, \varphi, \rho, \sigma ; e_{1}, e_{2}\right)\right| \\
& \leq 2^{\frac{1}{\wp}-2}\left\{\frac{\left(x^{\wp}-e_{1}^{\wp}\right)^{\varphi+1} \Theta_{2}^{1-\frac{1}{r}}\left(e_{1}\right)}{\mathcal{F}_{\rho, \varphi+1}^{\sigma}\left[\omega\left(e_{2}^{\wp}-e_{1}^{\wp}\right)^{\rho}\right]}\left[\Theta_{1}\left(e_{1}\right)\left|F^{\prime}(x)\right|^{r}+\left(\Theta_{2}\left(e_{1}\right)-\Theta_{1}\left(e_{1}\right)\right)\left|F^{\prime}\left(e_{1}\right)\right|^{r}\right]^{\frac{1}{r}}\right. \\
& \left.\quad+\frac{\left(e_{2}^{\wp}-x^{\wp}\right)^{\varphi+1} \Theta_{2}^{1-\frac{1}{r}}\left(e_{2}\right)}{\mathcal{F}_{\rho, \varphi+1}^{\sigma}\left[\omega\left(e_{2}^{\wp}-e_{1}^{\wp}\right)^{\rho}\right]}\left[\Theta_{1}\left(e_{2}\right)\left|F^{\prime}(x)\right|^{r}+\left(\Theta_{2}\left(e_{2}\right)-\Theta_{1}\left(e_{2}\right)\right)\left|F^{\prime}\left(e_{2}\right)\right|^{r}\right]^{\frac{1}{r}}\right\} .
\end{aligned}
$$

(b) For $\wp \in\left(\frac{1}{2}, 1\right]$, we have

$$
\begin{aligned}
& \left|\mathrm{Y}_{F}\left(\wp ; \alpha, \varphi, \rho, \sigma ; e_{1}, e_{2}\right)\right| \\
& \leq \frac{\left(x^{\wp}-e_{1}^{\wp}\right)^{\varphi+1} \Theta_{2}^{1-\frac{1}{r}}\left(e_{1}\right)}{\mathcal{F}_{\rho, \varphi+1}^{\sigma}\left[\omega\left(e_{2}^{\wp}-e_{1}^{\wp}\right)^{\rho}\right]}\left[\Theta_{1}\left(e_{1}\right)\left|F^{\prime}(x)\right|^{r}+\left(\Theta_{2}\left(e_{1}\right)-\Theta_{1}\left(e_{1}\right)\right)\left|F^{\prime}\left(e_{1}\right)\right|^{r}\right]^{\frac{1}{r}} \\
& \quad+\frac{\left(e_{2}^{\wp}-x^{\wp}\right)^{\varphi+1} \Theta_{2}^{1-\frac{1}{r}}\left(e_{2}\right)}{\mathcal{F}_{\rho, \varphi+1}^{\sigma}\left[\omega\left(e_{2}^{\wp}-e_{1}^{\wp}\right)^{\rho}\right]}\left[\Theta_{1}\left(e_{2}\right)\left|F^{\prime}(x)\right|^{r}+\left(\Theta_{2}\left(e_{2}\right)-\Theta_{1}\left(e_{2}\right)\right)\left|F^{\prime}\left(e_{2}\right)\right|^{r}\right]^{\frac{1}{r}},
\end{aligned}
$$


where $\Theta_{1}(\beta)$ and $\Theta_{2}(\beta)$ are defined by (29) and (30), respectively.

Proof. (a) For $\wp \in\left(0, \frac{1}{2}\right]$ and using Lemma 6, we have that

$$
\begin{aligned}
& \left|Y_{F}\left(\wp ; \alpha, \varphi, \rho, \sigma ; e_{1}, e_{2}\right)\right| \\
& \leq \frac{\left(x^{\wp}-e_{1}^{\wp}\right)^{\varphi+1}}{\mathcal{F}_{\rho, \varphi+1}^{\sigma}\left[\mathcal{\omega}\left(e_{2}^{\wp}-e_{1}^{\wp}\right)^{\rho}\right]} \int_{0}^{1}\left|\ell^{\varphi}-\alpha\right| \mathcal{F}_{\rho, \varphi+1}^{\sigma}\left[\mathfrak{\omega}\left(e_{2}^{\wp}-e_{1}^{\wp}\right)^{\rho}\left(\ell^{\rho}-\alpha\right)\right]\left(\ell x^{\wp}+(1-\ell) e_{1}^{\wp}\right)^{\frac{1}{\wp}-1}\left|F^{\prime}\left(\wp \sqrt{\ell x^{\wp}+(1-\ell) e_{1}^{\wp}}\right)\right| d \ell \\
& \quad-\frac{\left(e_{2}^{\wp}-x^{\wp}\right)^{\varphi+1}}{\mathcal{F}_{\rho, \varphi+1}^{\sigma}\left[\mathcal{\omega}\left(e_{2}^{\wp}-e_{1}^{\wp}\right)^{\rho}\right]} \int_{0}^{1}\left|\ell^{\varphi}-\alpha\right| \mathcal{F}_{\rho, \varphi+1}^{\sigma}\left[\omega\left(e_{2}^{\wp}-e_{1}^{\wp}\right)^{\rho}\left(\ell^{\rho}-\alpha\right)\right]\left(\ell x^{\wp}+(1-\ell) e_{2}^{\wp}\right)^{\frac{1}{\wp}-1}\left|F^{\prime}\left(\sqrt{\ell x^{\wp}+(1-\ell) e_{2}^{\wp}}\right)\right| d \ell .
\end{aligned}
$$

\section{Employing power-mean inequality, we have}

$$
\begin{aligned}
& \left|\mathrm{Y}_{F}\left(\wp ; \alpha, \varphi, \rho, \sigma ; e_{1}, e_{2}\right)\right| \\
& \leq \frac{\left(x^{\wp}-e_{1}^{\wp}\right)^{\varphi+1}}{\mathcal{F}_{\rho, \varphi+1}^{\sigma}\left[\omega\left(e_{2}^{\wp}-e_{1}^{\wp}\right)^{\rho}\right]} \\
& \times\left\{\begin{array}{c}
\left(\int_{0}^{1}\left|\ell^{\varphi}-\alpha\right| \mathcal{F}_{\rho, \varphi+1}^{\sigma}\left[\omega\left(e_{2}^{\wp}-e_{1}^{\wp}\right)^{\rho}\left(\ell^{\rho}-\alpha\right)\right]\left(\ell x^{\wp}+(1-\ell) e_{1}^{\wp}\right)^{\frac{1}{\wp}-1} d \ell\right)^{1-\frac{1}{r}} \\
\times\left(\int_{0}^{1}\left|\ell^{\varphi}-\alpha\right| \mathcal{F}_{\rho, \varphi+1}^{\sigma}\left[\omega\left(e_{2}^{\wp}-e_{1}^{\wp}\right)^{\rho}\left(\ell^{\rho}-\alpha\right)\right]\left(\ell x^{\wp}+(1-\ell) e_{1}^{\wp}\right)^{\frac{1}{\wp}-1}\left|F^{\prime}\left(\sqrt{\ell x^{\wp}+(1-\ell) e_{1}^{\wp}}\right)\right|^{r} d \ell\right)^{\frac{1}{r}}
\end{array}\right. \\
& -\frac{\left(e_{2}^{\wp}-x^{\wp}\right)^{\varphi+1}}{\mathcal{F}_{\rho, \varphi+1}^{\sigma}\left[\mathfrak{W}\left(e_{2}^{\wp}-e_{1}^{\wp}\right)^{\rho}\right]} \\
& \times\left\{\begin{array}{c}
\left(\int_{0}^{1}\left|\ell^{\varphi}-\alpha\right| \mathcal{F}_{\rho, \varphi+1}^{\sigma}\left[\omega\left(e_{2}^{\wp}-e_{1}^{\wp}\right)^{\rho}\left(\ell^{\rho}-\alpha\right)\right]\left(\ell x^{\wp}+(1-\ell) e_{2}^{\wp}\right)^{\frac{1}{\wp}-1} d \ell\right)^{1-\frac{1}{r}} \\
\times\left(\int_{0}^{1}\left|\ell^{\varphi}-\alpha\right| \mathcal{F}_{\rho, \varphi+1}^{\sigma}\left[\omega\left(e_{2}^{\wp}-e_{1}^{\wp}\right)^{\rho}\left(\ell^{\rho}-\alpha\right)\right]\left(\ell x^{\wp}+(1-\ell) e_{1}^{\wp}\right)^{\frac{1}{\zeta}-1}\left|F^{\prime}\left(\wp \sqrt{\ell x^{\wp}+(1-\ell) e_{2}^{\wp}}\right)\right|^{r} d \ell\right)^{\frac{1}{r}} .
\end{array}\right.
\end{aligned}
$$

Since $\left|F^{\prime}\right|^{r}$ is $\wp$-convex on $\Omega$, we have 


$$
\begin{aligned}
& \left|\mathrm{Y}_{F}\left(\wp ; \alpha, \varphi, \rho, \sigma ; e_{1}, e_{2}\right)\right| \\
& \leq \frac{\left(x^{\wp}-e_{1}^{\wp}\right)^{\varphi+1}}{\mathcal{F}_{\rho, \varphi+1}^{\sigma}\left[\omega\left(e_{2}^{\wp}-e_{1}^{\wp}\right)^{\rho}\right]} \\
& \times\left\{\begin{array}{c}
\left(\int_{0}^{1}\left|\ell^{\varphi}-\alpha\right| \mathcal{F}_{\rho, \varphi+1}^{\sigma}\left[\omega\left(e_{2}^{\wp}-e_{1}^{\wp}\right)^{\rho}\left(\ell^{\rho}-\alpha\right)\right]\left(\ell x^{\wp}+(1-\ell) e_{1}^{\wp}\right)^{\frac{1}{\wp}-1} d \ell\right)^{1-\frac{1}{r}} \\
\times\left(\int_{0}^{1}\left|\ell^{\varphi}-\alpha\right| \mathcal{F}_{\rho, \varphi+1}^{\sigma}\left[\omega\left(e_{2}^{\wp}-e_{1}^{\wp}\right)^{\rho}\left(\ell^{\rho}-\alpha\right)\right]\left(\ell x^{\wp}+(1-\ell) e_{1}^{\wp}\right)^{\frac{1}{\wp}-1}\left\{\ell\left|F^{\prime}(x)\right|^{r}+(1-\ell)\left|F^{\prime}\left(e_{1}\right)\right|^{r}\right\} d \ell\right)^{\frac{1}{r}}
\end{array}\right. \\
& -\frac{\left(e_{2}^{\wp}-x^{\wp}\right)^{\varphi+1}}{\mathcal{F}_{\rho, \varphi+1}^{\sigma}\left[\omega\left(e_{2}^{\wp}-e_{1}^{\wp}\right)^{\rho}\right]} \\
& \times\left\{\begin{array}{c}
\left(\int_{0}^{1}\left|\ell^{\varphi}-\alpha\right| \mathcal{F}_{\rho, \varphi+1}^{\sigma}\left[\omega\left(e_{2}^{\wp}-e_{1}^{\wp}\right)^{\rho}\left(\ell^{\rho}-\alpha\right)\right]\left(\ell x^{\wp}+(1-\ell) e_{2}^{\wp}\right)^{\frac{1}{\wp}-1} d \ell\right)^{1-\frac{1}{r}} \\
\times\left(\int_{0}^{1}\left|\ell^{\varphi}-\alpha\right| \mathcal{F}_{\rho, \varphi+1}^{\sigma}\left[\omega\left(e_{2}^{\wp}-e_{1}^{\wp}\right)^{\rho}\left(\ell^{\rho}-\alpha\right)\right]\left(\ell x^{\wp}+(1-\ell) e_{1}^{\wp}\right)^{\frac{1}{\wp}-1}\left\{\ell\left|F^{\prime}(x)\right|^{r}+(1-\ell)\left|F^{\prime}\left(e_{2}\right)\right|^{r}\right\} d \ell\right)^{\frac{1}{r}}
\end{array}\right. \\
& \leq \frac{\left(x^{\wp}-e_{1}^{\wp}\right)^{\varphi+1}}{\mathcal{F}_{\rho, \varphi+1}^{\sigma}\left[\omega\left(e_{2}^{\wp}-e_{1}^{\wp}\right)^{\rho}\right]} \\
& \times\left\{\begin{array}{c}
\left(\int_{0}^{1}\left|\ell^{\varphi}-\alpha\right| \mathcal{F}_{\rho, \varphi+1}^{\sigma}\left[\omega\left(e_{2}^{\wp}-e_{1}^{\wp}\right)^{\rho}\left(\ell^{\rho}-\alpha\right)\right]\left(\ell x^{\wp}+(1-\ell) e_{1}^{\wp}\right)^{\frac{1}{\wp}-1} d \ell\right)^{1-\frac{1}{r}} \\
\times\left(\left|F^{\prime}(x)\right|^{r} \int_{0}^{1} \ell\left|\ell^{\varphi}-\alpha\right| \mathcal{F}_{\rho, \varphi+1}^{\sigma}\left[\omega\left(e_{2}^{\wp}-e_{1}^{\wp}\right)^{\rho}\left(\ell^{\rho}-\alpha\right)\right]\left(\ell x^{\wp}+(1-\ell) e_{1}^{\wp}\right)^{\frac{1}{\wp}-1} d \ell\right. \\
\left.+\left|F^{\prime}\left(e_{1}\right)\right|^{r} \int_{0}^{1}(1-\ell)\left|\ell^{\varphi}-\alpha\right| \mathcal{F}_{\rho, \varphi+1}^{\sigma}\left[\omega\left(e_{2}^{\wp}-e_{1}^{\wp}\right)^{\rho}\left(\ell^{\rho}-\alpha\right)\right]\left(\ell x^{\wp}+(1-\ell) e_{1}^{\wp}\right)^{\frac{1}{\wp}-1} d \ell\right)^{\frac{1}{r}}
\end{array}\right. \\
& -\frac{\left(e_{2}^{\wp}-x^{\wp}\right)^{\varphi+1}}{\mathcal{F}_{\rho, \varphi+1}^{\sigma}\left[\omega\left(e_{2}^{\wp}-e_{1}^{\wp}\right)^{\rho}\right]} \\
& \times\left\{\begin{array}{c}
\left(\int_{0}^{1}\left|\ell^{\varphi}-\alpha\right| \mathcal{F}_{\rho, \varphi+1}^{\sigma}\left[\omega\left(e_{2}^{\wp}-e_{1}^{\wp}\right)^{\rho}\left(\ell^{\rho}-\alpha\right)\right]\left(\ell x^{\wp}+(1-\ell) e_{2}^{\wp}\right)^{\frac{1}{\wp}-1} d \ell\right)^{1-\frac{1}{r}} \\
\times\left(\left|F^{\prime}(x)\right|^{r} \int_{0}^{1} \ell\left|\ell^{\varphi}-\alpha\right| \mathcal{F}_{\rho, \varphi+1}^{\sigma}\left[\omega\left(e_{2}^{\wp}-e_{1}^{\wp}\right)^{\rho}\left(\ell^{\rho}-\alpha\right)\right]\left(\ell x^{\wp}+(1-\ell) e_{2}^{\wp}\right)^{\frac{1}{\wp}-1} d \ell\right. \\
\left.+\left|F^{\prime}\left(e_{2}\right)\right|^{r} \int_{0}^{1}(1-\ell)\left|\ell^{\varphi}-\alpha\right| \mathcal{F}_{\rho, \varphi+1}^{\sigma}\left[\omega\left(e_{2}^{\wp}-e_{1}^{\wp}\right)^{\rho}\left(\ell^{\rho}-\alpha\right)\right]\left(\ell x^{\wp}+(1-\ell) e_{2}^{\wp}\right)^{\frac{1}{\wp}-1} d \ell\right)^{\frac{1}{r}} .
\end{array}\right.
\end{aligned}
$$

Combining (40), (35) and (36), we get the desired inequality (38).

To prove $(b)$, let $\wp \in\left(\frac{1}{2}, 1\right]$, then we get the required inequality in (39) by employing the inequality (14). So, this completes the proof.

Corollary 2. Theorem 3 with $\left|F^{\prime}\right| \leq \mathcal{M}$ reduces to

(a) For $\wp \in\left(0, \frac{1}{2}\right]$, the following inequality holds:

$$
\begin{aligned}
& \left|\mathrm{Y}_{F}\left(\wp ; \alpha, \varphi, \rho, \sigma ; e_{1}, e_{2}\right)\right| \\
& \leq 2^{\frac{1}{\wp}-2} \mathcal{M}\left\{\frac{\left(x^{\wp}-e_{1}^{\wp}\right)^{\varphi+1}}{\mathcal{F}_{\rho, \varphi+1}^{\sigma}\left[\omega\left(e_{2}^{\wp}-e_{1}^{\wp}\right)^{\rho}\right]} \Theta_{2}\left(e_{1}\right)+\frac{\left(e_{2}^{\wp}-x^{\wp}\right)^{\varphi+1}}{\mathcal{F}_{\rho, \varphi+1}^{\sigma}\left[\omega\left(e_{2}^{\wp}-e_{1}^{\wp}\right)^{\rho}\right]} \Theta_{2}\left(e_{2}\right)\right\} .
\end{aligned}
$$


(b) For $\wp \in\left(\frac{1}{2}, 1\right]$, the following inequality holds:

$$
\begin{aligned}
& \left|Y_{F}\left(\wp ; \alpha, \varphi, \rho, \sigma ; e_{1}, e_{2}\right)\right| \\
& \leq \mathcal{M}\left\{\frac{\left(x^{\wp}-e_{1}^{\wp}\right)^{\varphi+1}}{\mathcal{F}_{\rho, \varphi+1}^{\sigma}\left[\omega\left(e_{2}^{\wp}-e_{1}^{\wp}\right)^{\rho}\right]} \Theta_{2}\left(e_{1}\right)+\frac{\left(e_{2}^{\wp}-x^{\wp}\right)^{\varphi+1}}{\mathcal{F}_{\rho, \varphi+1}^{\sigma}\left[\omega\left(e_{2}^{\wp}-e_{1}^{\wp}\right)^{\rho}\right]} \Theta_{2}\left(e_{2}\right)\right\} .
\end{aligned}
$$

Theorem 4. For $\wp \leq 0, \alpha \in[0,1], s, r>1$ and $r^{-1}+s^{-1}=1$. Let there be a differentiable function $F: \Omega \subset(0, \infty) \rightarrow \mathbb{R}$ on $\Omega^{\circ}$ with $e_{1}<e_{2}$ such that $F^{\prime} \in L_{1}\left(\left[e_{1}, e_{2}\right]\right)$. If $\left|F^{\prime}\right|^{r}$ is $\wp$-convex on $\Omega$, then for all $x \in\left(e_{1}, e_{2}\right)$, the following inequality holds:

$$
\begin{aligned}
& \left|\mathrm{Y}_{F}\left(\wp ; \alpha, \varphi, \rho, \sigma ; e_{1}, e_{2}\right)\right| \\
& \leq\left\{\frac{\left(x^{\wp}-e_{1}^{\wp}\right)^{\varphi+1} \Phi_{3}^{\frac{1}{s}}\left(e_{1}\right)}{\mathcal{F}_{\rho, \varphi+1}^{\sigma}\left[\omega\left(e_{2}^{\wp}-e_{1}^{\wp}\right)^{\rho}\right]}\left[\Phi_{1}(r)\left|F^{\prime}(x)\right|^{r}+\Phi_{2}(q)\left|F^{\prime}\left(e_{1}\right)\right|^{r}\right]^{\frac{1}{r}}\right. \\
& \left.\quad+\frac{\left(e_{2}^{\wp}-x^{\wp}\right)^{\varphi+1} \Phi_{3}^{\frac{1}{s}}\left(e_{2}\right)}{\mathcal{F}_{\rho, \varphi+1}^{\sigma}\left[\omega\left(e_{2}^{\wp}-e_{1}^{\wp}\right)^{\rho}\right]}\left[\Phi_{1}(r)\left|F^{\prime}(x)\right|^{r}+\Phi_{2}(r)\left|F^{\prime}\left(e_{2}\right)\right|^{r}\right]^{\frac{1}{r}}\right\},
\end{aligned}
$$

where

$$
\begin{aligned}
\Phi_{1}(r) \quad:= & \left(\sum_{\kappa=0}^{\infty} \frac{\sigma(\kappa) \omega^{\kappa}\left(e_{2}^{\wp}-e_{1}^{\wp}\right)}{\Gamma(\rho \kappa+\varphi+1)}\right)^{r}\left[\frac{4 \alpha^{\frac{r \varphi+r \rho \kappa+2}{\varphi}}}{(r \rho \kappa+2)(r \rho \kappa+r \varphi+2)}+\frac{1}{r \rho \kappa+r \varphi+2}-\frac{\alpha^{r}}{r \rho \kappa+2}\right], \\
\Phi_{2}(r) \quad:= & \left(\sum_{\kappa=0}^{\infty} \frac{\sigma(\kappa) \omega^{\kappa}\left(e_{2}^{\wp}-e_{1}^{\wp}\right)}{\Gamma(\rho \kappa+\varphi+1)}\right)^{r}\left[\frac{2 r \varphi \alpha^{\frac{r \varphi+r \rho \kappa+1}{\varphi}}}{(r \rho \kappa+1)(r \rho \kappa+r \varphi+1)}-\frac{4 \alpha^{\frac{r \varphi+r \rho \kappa+2}{\varphi}}}{(r \rho \kappa+2)(r \rho \kappa+r \varphi+2)}\right. \\
& \left.+\frac{1}{(r \rho \kappa+r \varphi+1)(r \rho \kappa+r \varphi+2)}-\frac{\alpha^{r}}{(r \rho \kappa+2)(r \rho \kappa+1)}\right], \\
\Phi_{3}(\beta) \quad:= & \frac{\wp}{x^{\wp}-\beta^{\wp}}\left[\frac{x^{(s-1)(1-\wp)+1}-\beta^{(s-1)(1-\wp)+1}}{(s-1)(1-\wp)+1}\right], \beta \in\left\{e_{1}, e_{2}\right\} .
\end{aligned}
$$

Proof. By means of Lemma 6 and applying absolute, we have

$$
\begin{aligned}
& \left|\mathrm{Y}_{F}\left(\wp ; \alpha, \varphi, \rho, \sigma ; e_{1}, e_{2}\right)\right| \\
& \leq \frac{\left(x^{\wp}-e_{1}^{\wp}\right)^{\varphi+1}}{\mathcal{F}_{\rho, \varphi+1}^{\sigma}\left[\omega\left(e_{2}^{\wp}-e_{1}^{\wp}\right)^{\rho}\right]} \int_{0}^{1}\left|\ell^{\varphi}-\alpha\right| \mathcal{F}_{\rho, \varphi+1}^{\sigma}\left[\omega\left(e_{2}^{\wp}-e_{1}^{\wp}\right)^{\rho}\left(\ell^{\rho}-\alpha\right)\right]\left(\ell x^{\wp}+(1-\ell) e_{1}^{\wp}\right)^{\frac{1}{\wp}-1}\left|F^{\prime}\left(\wp \sqrt{\ell x^{\wp}+(1-\ell) e_{1}^{\wp}}\right)\right| d \ell \\
& \quad-\frac{\left(e_{2}^{\wp}-x^{\wp}\right)^{\varphi+1}}{\mathcal{F}_{\rho, \varphi+1}^{\sigma}\left[\omega\left(e_{2}^{\wp}-e_{1}^{\wp}\right)^{\rho}\right]} \int_{0}^{1}\left|\ell^{\varphi}-\alpha\right| \mathcal{F}_{\rho, \varphi+1}^{\sigma}\left[\omega\left(e_{2}^{\wp}-e_{1}^{\wp}\right)^{\rho}\left(\ell^{\rho}-\alpha\right)\right]\left(\ell x^{\wp}+(1-\ell) e_{2}^{\wp}\right)^{\frac{1}{\wp}-1}\left|F^{\prime}\left(\sqrt{\ell x^{\wp}+(1-\ell) e_{2}^{\wp}}\right)\right| d \ell .
\end{aligned}
$$

Employing Hölder inequality, we have 


$$
\begin{aligned}
& \left|\mathrm{Y}_{F}\left(\wp ; \alpha, \varphi, \rho, \sigma ; e_{1}, e_{2}\right)\right| \\
& \leq \frac{\left(x^{\wp}-e_{1}^{\wp}\right)^{\varphi+1}}{\mathcal{F}_{\rho, \varphi+1}^{\sigma}\left[\mathscr{\omega}\left(e_{2}^{\wp}-e_{1}^{\wp}\right)^{\rho}\right]} \\
& \times\left(\int_{0}^{1}\left(\ell x^{\wp}+(1-\ell) e_{1}^{\wp}\right)^{\frac{s}{\xi}-s} d \ell\right)^{\frac{1}{s}}\left(\int_{0}^{1}\left|\ell^{\varphi}-\alpha\right|^{r}\left(\mathcal{F}_{\rho, \varphi+1}^{\sigma}\left[\omega\left(e_{2}^{\wp}-e_{1}^{\wp}\right)^{\rho}\left(\ell^{\rho}-\alpha\right)\right]\right)^{r}\left|F^{\prime}\left(\wp \sqrt{\ell x^{\wp}+(1-\ell) e_{1}^{\wp}}\right)\right|^{r} d \ell\right)^{\frac{1}{r}} \\
& -\frac{\left(e_{2}^{\wp}-x^{\wp}\right)^{\varphi+1}}{\mathcal{F}_{\rho, \varphi+1}^{\sigma}\left[\mathfrak{\omega}\left(e_{2}^{\wp}-e_{1}^{\wp}\right)^{\rho}\right]} \\
& \times\left(\int_{0}^{1}\left(\ell x^{\wp}+(1-\ell) e_{2}^{\wp}\right)^{\frac{s}{\zeta}-s} d \ell\right)^{\frac{1}{s}}\left(\int_{0}^{1}\left|\ell^{\varphi}-\alpha\right|^{r}\left(\mathcal{F}_{\rho, \varphi+1}^{\sigma}\left[\omega\left(e_{2}^{\wp}-e_{1}^{\wp}\right)^{\rho}\left(\ell^{\rho}-\alpha\right)\right]\right)^{r}\left|F^{\prime}\left(\wp \sqrt{\ell x^{\wp}+(1-\ell) e_{2}^{\wp}}\right)\right|^{r} d \ell\right)^{\frac{1}{r}} .
\end{aligned}
$$

Since $\left|F^{\prime}\right|^{r}$ is $\wp$-convex on $\Omega$, we have

$$
\begin{aligned}
& \left|Y_{F}\left(\wp ; \alpha, \varphi, \rho, \sigma ; e_{1}, e_{2}\right)\right| \\
& \leq \frac{\left(x^{\wp}-e_{1}^{\wp}\right)^{\varphi+1}}{\mathcal{F}_{\rho, \varphi+1}^{\sigma}\left[\omega\left(e_{2}^{\wp}-e_{1}^{\wp}\right)^{\rho}\right]} \Phi_{3}(\beta)^{\frac{1}{s}}\left(\int_{0}^{1}\left|\ell^{\varphi}-\alpha\right|^{r}\left(\mathcal{F}_{\rho, \varphi+1}^{\sigma}\left[\omega\left(e_{2}^{\wp}-e_{1}^{\wp}\right)^{\rho}\left(\ell^{\rho}-\alpha\right)\right]\right)^{r}\left[\ell\left|F^{\prime}(x)\right|^{r}+(1-\ell)\left|F^{\prime}\left(e_{1}\right)\right|^{r}\right] d \ell\right)^{\frac{1}{r}} \\
& \quad-\frac{\left(e_{2}^{\wp}-x^{\wp}\right)^{\varphi+1}}{\mathcal{F}_{\rho, \varphi+1}^{\sigma}\left[\omega\left(e_{2}^{\wp}-e_{1}^{\wp}\right) \rho\right]} \Phi_{3}(\beta)^{\frac{1}{s}}\left(\int_{0}^{1}\left|\ell^{\varphi}-\alpha\right|^{r}\left(\mathcal{F}_{\rho, \varphi+1}^{\sigma}\left[\omega\left(e_{2}^{\wp}-e_{1}^{\wp}\right)^{\rho}\left(\ell^{\rho}-\alpha\right)\right]\right)^{r}\left[\ell\left|F^{\prime}(x)\right|^{r}+(1-\ell)\left|F^{\prime}\left(e_{2}\right)\right|^{r}\right] d \ell\right)^{\frac{1}{r}} \\
& =\frac{\left(x^{\wp}-e_{1}^{\wp}\right)^{\varphi+1}}{\mathcal{F}_{\rho, \varphi+1}^{\sigma}\left[\omega\left(e_{2}^{\wp}-e_{1}^{\wp}\right)^{\rho}\right]} \Phi_{3}(\beta)^{\frac{1}{s}}\left\{\begin{array}{c}
\left(\left|F^{\prime}(x)\right|^{r} \int_{0}^{1} \ell\left|\ell^{\varphi}-\alpha\right| \mathcal{F}_{\rho, \varphi+1}^{\sigma}\left[\omega\left(e_{2}^{\wp}-e_{1}^{\wp}\right)^{\rho}\left(\ell^{\rho}-\alpha\right)\right] d \ell\right. \\
\left.+\left|F^{\prime}\left(e_{1}\right)\right|^{r} \int_{0}^{1}(1-\ell)\left|\ell^{\varphi}-\alpha\right| \mathcal{F}_{\rho, \varphi+1}^{\sigma}\left[\omega\left(e_{2}^{\wp}-e_{1}^{\wp}\right)^{\rho}\left(\ell^{\rho}-\alpha\right)\right] d \ell\right)^{\frac{1}{r}}
\end{array}\right. \\
& -\frac{\left(e_{2}^{\wp}-x^{\wp}\right)^{\varphi+1}}{\mathcal{F}_{\rho, \varphi+1}^{\sigma}\left[\omega\left(e_{2}^{\wp}-e_{1}^{\wp}\right)^{\rho}\right]} \Phi_{3}(\beta)^{\frac{1}{s}}\left\{\begin{array}{c}
\left(\left|F^{\prime}(x)\right|^{r} \int_{0}^{1} \ell\left|\ell^{\varphi}-\alpha\right| \mathcal{F}_{\rho, \varphi+1}^{\sigma}\left[\omega\left(e_{2}^{\wp}-e_{1}^{\wp}\right)^{\rho}\left(\ell^{\rho}-\alpha\right)\right] d \ell\right. \\
\left.+\left|F^{\prime}\left(e_{2}\right)\right|^{r} \int_{0}^{1}(1-\ell)\left|\ell^{\varphi}-\alpha\right| \mathcal{F}_{\rho, \varphi+1}^{\sigma}\left[\omega\left(e_{2}^{\wp}-e_{1}^{\wp}\right)^{\rho}\left(\ell^{\rho}-\alpha\right)\right] d \ell\right)^{\frac{1}{r}} .
\end{array}\right.
\end{aligned}
$$

Considering the inequality $\left(\mu_{1}-\mu_{2}\right)^{c} \leq \mu_{1}^{c}-\mu_{2}^{c}$ for any $0 \leq \mu_{2}<\mu_{1}$ and $c \geq 1$, it gives that

$$
\begin{aligned}
& \int_{0}^{1} \ell\left|\ell^{\varphi}-\alpha\right|^{r}\left(\mathcal{F}_{\rho, \varphi+1}^{\sigma}\left[\omega\left(e_{2}^{\wp}-e_{1}^{\wp}\right)^{\rho}\left(\ell^{\rho}-\alpha\right)\right]\right)^{r} d \ell \\
& =\left(\sum_{\kappa=0}^{\infty} \frac{\sigma(\kappa) \omega^{\kappa}\left(e_{2}^{\wp}-e_{1}^{\wp}\right)}{\Gamma(\rho \kappa+\varphi+1)}\right)^{r}\left[\int_{0}^{\alpha^{1 / \varphi}} \ell^{r \rho \kappa+1}\left(\alpha-\ell^{\varphi}\right)^{r} d \ell+\int_{\alpha^{1 / \varphi}}^{1} \ell^{r \rho \kappa+1}\left(\ell^{\varphi}-\alpha\right)^{r} d \ell\right] \\
& \leq\left(\sum_{\kappa=0}^{\infty} \frac{\sigma(\kappa) \omega^{\kappa}\left(e_{2}^{\wp}-e_{1}^{\wp}\right)}{\Gamma(\rho \kappa+\varphi+1)}\right)^{r}\left[\int_{0}^{\alpha^{1 / \varphi}} \ell^{r \rho \kappa+1}\left(\alpha^{r}-\ell^{r \varphi}\right) d \ell+\int_{\alpha^{1 / \varphi}}^{1} \ell^{r \rho \kappa+1}\left(\ell^{r \varphi}-\alpha^{r}\right) d \ell\right] \\
& =\left(\sum_{\kappa=0}^{\infty} \frac{\sigma(\kappa) \omega^{\kappa}\left(e_{2}^{\wp}-e_{1}^{\wp}\right)}{\Gamma(\rho \kappa+\varphi+1)}\right)^{r}\left[\frac{4 \alpha^{\frac{r \varphi+r \rho \kappa+2}{\varphi}}}{(r \rho \kappa+2)(r \rho \kappa+r \varphi+2)}+\frac{1}{r \rho \kappa+r \varphi+2}-\frac{\alpha^{r}}{r \rho \kappa+2}\right] .
\end{aligned}
$$




$$
\begin{aligned}
& \int_{0}^{1}(1-\ell)\left|\ell^{\varphi}-\alpha\right|^{r}\left(\mathcal{F}_{\rho, \varphi+1}^{\sigma}\left[\mathcal{\omega}\left(e_{2}^{\wp}-e_{1}^{\wp}\right)^{\rho}\left(\ell^{\rho}-\alpha\right)\right]\right)^{r} d \ell \\
& =\left(\sum_{\kappa=0}^{\infty} \frac{\sigma(\kappa) \omega^{\kappa}\left(e_{2}^{\wp}-e_{1}^{\wp}\right)}{\Gamma(\rho \kappa+\varphi+1)}\right)^{r}\left[\int_{0}^{\alpha^{1 / \varphi}}(1-\ell)^{r \rho \kappa+1}\left(\alpha-\ell^{\varphi}\right)^{r} d \ell+\int_{\alpha^{1 / \varphi}}^{1}(1-\ell)^{r \rho \kappa+1}\left(\ell^{\varphi}-\alpha\right)^{r} d \ell\right] \\
& \leq\left(\sum_{\kappa=0}^{\infty} \frac{\sigma(\kappa) \omega^{\kappa}\left(e_{2}^{\wp}-e_{1}^{\wp}\right)}{\Gamma(\rho \kappa+\varphi+1)}\right)^{r}\left[\int_{0}^{\alpha^{1 / \varphi}} \ell^{\rho \kappa+1}\left(\alpha^{r}-\ell^{r \varphi}\right) d \ell+\int_{\alpha^{1 / \varphi}}^{1} \ell^{\rho \kappa+1}\left(\ell^{r \varphi}-\alpha^{r}\right) d \ell\right] \\
& =\left(\sum_{\kappa=0}^{\infty} \frac{\sigma(\kappa) \mathcal{\omega}^{\kappa}\left(e_{2}^{\wp}-e_{1}^{\wp}\right)}{\Gamma(\rho \kappa+\varphi+1)}\right)^{r}\left[\frac{2 r \varphi \alpha^{\frac{r \varphi+r \rho \kappa+1}{\varphi}}}{(r \rho \kappa+1)(r \rho \kappa+r \varphi+1)}-\frac{4 \alpha^{\frac{r \varphi+r \rho \kappa+2}{\varphi}}}{(r \rho \kappa+2)(r \rho \kappa+r \varphi+2)}\right. \\
& \left.\quad+\frac{1}{(r \rho \kappa+r \varphi+1)(r \rho \kappa+r \varphi+2)}-\frac{\alpha^{r}}{(r \rho \kappa+2)(r \rho \kappa+1)}\right] .
\end{aligned}
$$

Using the fact that

$$
\begin{aligned}
\Phi_{3}(\beta) \quad & :=\int_{0}^{1}\left(\ell x^{\wp}+(1-\ell) e_{1}^{\wp}\right)^{\frac{s}{\wp}-s} d \ell \\
& =\frac{\wp}{x^{\wp}-\beta \wp}\left[\frac{x^{(s-1)(1-\wp)+1}-\beta^{(s-1)(1-\wp)+1}}{(s-1)(1-\wp)+1}\right], \beta \in\left\{e_{1}, e_{2}\right\} .
\end{aligned}
$$

So, this completes the proof.

Corollary 3. Theorem 4 with $\left|F^{\prime}\right| \leq \mathcal{M}$ reduces to

$$
\begin{aligned}
& \left|\mathrm{Y}_{F}\left(\wp ; \alpha, \varphi, \rho, \sigma ; e_{1}, e_{2}\right)\right| \\
& \leq \mathcal{M}\left(\Phi_{1}(r)+\Phi_{2}(r)\right)^{\frac{1}{r}}\left[\frac{\left(x^{\wp}-e_{1}^{\wp}\right)^{\varphi+1} \Phi_{3}^{\frac{1}{s}}\left(e_{1}\right)+\left(e_{2}^{\wp}-x^{\wp}\right)^{\varphi+1} \Phi_{3}^{\frac{1}{s}}\left(e_{2}\right)}{\mathcal{F}_{\rho, \varphi+1}^{\sigma}\left[\omega\left(e_{2}^{\wp}-e_{1}^{\wp}\right)^{\rho}\right]}\right] .
\end{aligned}
$$

Theorem 5. For $r>1,0<\wp \leq 1,\left(\frac{1}{2}\right)^{\varphi}<\alpha \leq 1$ and let there be a differentiable function $F: \Omega \subset(0, \infty) \rightarrow \mathbb{R}$ on $\Omega^{\circ}$ with $e_{1}<e_{2}$ such that $F^{\prime} \in L_{1}\left(\left[e_{1}, e_{2}\right]\right)$. If $\left|F^{\prime}\right|^{r}$ is $\wp$-convex on $\Omega$, then for all $x \in\left(e_{1}, e_{2}\right)$, the following inequalities holds:

(a) For $\wp \in\left(0, \frac{1}{1+\frac{1}{s}}\right]$, we have

$$
\begin{aligned}
& \left|\mathrm{Y}_{F}\left(\wp ; \alpha, \varphi, \rho, \sigma ; e_{1}, e_{2}\right)\right| \\
& \leq 2^{\frac{s}{\varphi}-s-1}\left\{\frac{\left(x^{\wp}-e_{1}^{\wp}\right)^{\varphi+1} \Psi^{\frac{1}{s}}\left(e_{1}\right)}{\mathcal{F}_{\rho, \varphi+1}^{\sigma}\left[\omega\left(e_{2}^{\wp}-e_{1}^{\wp}\right)^{\rho}\right]}\left[\Phi_{1}(1)\left|F^{\prime}(x)\right|^{r}+\Phi_{2}(1)\left|F^{\prime}\left(e_{1}\right)\right|^{r}\right]^{\frac{1}{r}}\right. \\
& \left.\quad+\frac{\left(e_{2}^{\wp}-x^{\wp}\right)^{\varphi+1} \Psi^{\frac{1}{s}}\left(e_{2}\right)}{\mathcal{F}_{\rho, \varphi+1}^{\sigma}\left[\omega\left(e_{2}^{\wp}-e_{1}^{\wp}\right)^{\rho}\right]}\left[\Phi_{1}(1)\left|F^{\prime}(x)\right|^{r}+\Phi_{2}(1)\left|F^{\prime}\left(e_{2}\right)\right|^{r}\right]^{\frac{1}{r}}\right\} .
\end{aligned}
$$

(b) For $\wp \in\left(\frac{1}{1+\frac{1}{s+1}}, 1\right]$, we have 


$$
\begin{aligned}
& \left|\mathrm{Y}_{F}\left(\wp ; \alpha, \varphi, \rho, \sigma ; e_{1}, e_{2}\right)\right| \\
& \leq \frac{\left(x^{\wp}-e_{1}^{\wp}\right)^{\varphi+1} \Psi^{\frac{1}{s}}\left(e_{1}\right)}{\mathcal{F}_{\rho, \varphi+1}^{\sigma}\left[\mathscr{\omega}\left(e_{2}^{\wp}-e_{1}^{\wp}\right)^{\rho}\right]}\left[\Phi_{1}(1)\left|F^{\prime}(x)\right|^{r}+\Phi_{2}(1)\left|F^{\prime}\left(e_{1}\right)\right|^{r}\right]^{\frac{1}{r}} \\
& \quad+\frac{\left(e_{2}^{\wp}-x^{\wp}\right)^{\varphi+1} \Psi^{\frac{1}{s}}\left(e_{2}\right)}{\mathcal{F}_{\rho, \varphi+1}^{\sigma}\left[\omega\left(e_{2}^{\wp}-e_{1}^{\wp}\right)^{\rho}\right]}\left[\Phi_{1}(1)\left|F^{\prime}(x)\right|^{r}+\Phi_{2}(1)\left|F^{\prime}\left(e_{2}\right)\right|^{r}\right]^{\frac{1}{r}} .
\end{aligned}
$$

where

$$
\begin{aligned}
\Phi_{1}(1) \quad:= & \sum_{\kappa=0}^{\infty} \frac{\sigma(\kappa) \omega^{\kappa}\left(e_{2}^{\wp}-e_{1}^{\wp}\right)}{\Gamma(\rho \kappa+\varphi+1)}\left[\frac{4 \alpha^{\frac{\varphi+\rho \kappa+2}{\varphi}}}{(\rho \kappa+2)(\rho \kappa+\varphi+2)}+\frac{1}{\rho \kappa+\varphi+2}-\frac{\alpha}{\rho \kappa+2}\right], \\
\Phi_{2}(1) \quad:= & \sum_{\kappa=0}^{\infty} \frac{\sigma(\kappa) \omega^{\kappa}\left(e_{2}^{\wp}-e_{1}^{\wp}\right)}{\Gamma(\rho \kappa+\varphi+1)}\left[\frac{2 \varphi \alpha^{\frac{\varphi+\rho \kappa+1}{\varphi}}}{(\rho \kappa+1)(\rho \kappa+\varphi+1)}-\frac{4 \alpha^{\frac{\varphi+\rho \kappa+2}{\varphi}}}{(\rho \kappa+2)(\rho \kappa+\varphi+2)}\right. \\
& \left.+\frac{1}{(\rho \kappa+\varphi+1)(\rho \kappa+\varphi+2)}-\frac{\alpha}{(\rho \kappa+2)(\rho \kappa+1)}\right]
\end{aligned}
$$

and

$$
\begin{aligned}
\Psi(\beta):= & \sum_{\kappa=0}^{\infty} \frac{\sigma(\kappa) \omega^{\kappa}\left(e_{2}^{\wp}-e_{1}^{\wp}\right)}{\Gamma(\rho \kappa+\varphi+1)}\left[x ^ { s - s \wp } \left\{\frac{2 \alpha^{\frac{\wp \rho \kappa+s-s \wp+2 \wp}{\varphi}}}{\rho \kappa+\frac{s}{\wp}-s+1}-\frac{2 \alpha^{\frac{\wp \rho \kappa+\wp \varphi+s-s \wp+\wp}{\varphi}}}{\varphi+\rho \kappa+\frac{s}{\wp}-s+1}+\frac{1}{\varphi+\rho \kappa+\frac{s}{\wp}-s+1}\right.\right. \\
& -\frac{\alpha}{\rho \kappa+\frac{s}{\wp}-s+1}+\beta^{s-\varsigma \wp}\left[\alpha\left(\mathbb{B}_{\alpha^{1 / \varphi}}(\rho \kappa+1, s / \wp-s+1)-\mathbb{B}_{1-\alpha^{1 / \varphi}}(\rho \kappa+1, s / \wp-s+1)\right)\right. \\
& \left.\left.\left.-\mathbb{B}_{\alpha^{1 / \varphi}}(\varphi+\rho \kappa+1, s / \wp-s+1)+\mathbb{B}_{1-\alpha^{1 / \varphi}}(\varphi+\rho \kappa+1, s / \wp-s+1)\right)\right]\right\} .
\end{aligned}
$$

Proof. (a) For $\wp \in\left(0, \frac{1}{1+\frac{1}{s}}\right]$ and using Lemma 6, we have that

$$
\begin{aligned}
& \left|\mathrm{Y}_{F}\left(\wp ; \alpha, \varphi, \rho, \sigma ; e_{1}, e_{2}\right)\right| \\
& \leq \frac{\left(x^{\wp}-e_{1}^{\wp}\right)^{\varphi+1}}{\mathcal{F}_{\rho, \varphi+1}^{\sigma}\left[\mathcal{\omega}\left(e_{2}^{\wp}-e_{1}^{\wp}\right)^{\rho}\right]} \int_{0}^{1}\left|\ell^{\varphi}-\alpha\right| \mathcal{F}_{\rho, \varphi+1}^{\sigma}\left[\omega\left(e_{2}^{\wp}-e_{1}^{\wp}\right)^{\rho}\left(\ell^{\rho}-\alpha\right)\right]\left(\ell x^{\wp}+(1-\ell) e_{1}^{\wp}\right)^{\frac{1}{\wp}-1}\left|F^{\prime}\left(\wp \sqrt{\ell x^{\wp}+(1-\ell) e_{1}^{\wp}}\right)\right| d \ell \\
& \quad-\frac{\left(e_{2}^{\wp}-x^{\wp}\right)^{\varphi+1}}{\mathcal{F}_{\rho, \varphi+1}^{\sigma}\left[\omega\left(e_{2}^{\wp}-e_{1}^{\wp}\right)^{\rho}\right]} \int_{0}^{1}\left|\ell^{\varphi}-\alpha\right| \mathcal{F}_{\rho, \varphi+1}^{\sigma}\left[\omega\left(e_{2}^{\wp}-e_{1}^{\wp}\right)^{\rho}\left(\ell^{\rho}-\alpha\right)\right]\left(\ell x^{\wp}+(1-\ell) e_{2}^{\wp}\right)^{\frac{1}{\wp}-1}\left|F^{\prime}\left(\sqrt{\ell x^{\wp}+(1-\ell) e_{2}^{\wp}}\right)\right| d \ell .
\end{aligned}
$$

Employing the weighted Hölder's inequality (see [36]),

$$
\left|\int_{C} F(x) Q(x) P(x) d x\right| \leq\left(\int_{C}|F(x)|^{s} P(x) d x\right)^{\frac{1}{s}}\left(\int_{C}|Q(x)|^{r} P(x) d x\right)^{\frac{1}{r}}
$$

for $r>1, s^{-1}+r^{-1}=1$ and a non-negative mapping $P$ on $I$ has finite integral representation, we have 


$$
\begin{aligned}
& \left|Y_{F}\left(\wp ; \alpha, \varphi, \rho, \sigma ; e_{1}, e_{2}\right)\right|
\end{aligned}
$$

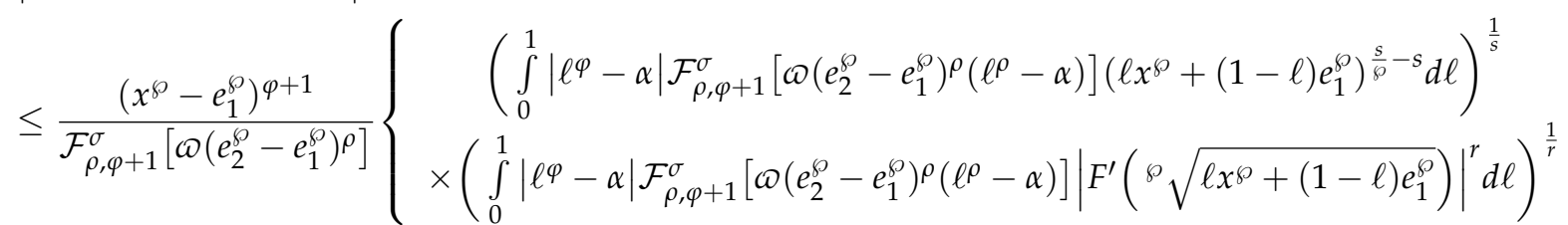

$$
\begin{aligned}
& -\frac{\left(e_{2}^{\wp}-x^{\wp}\right)^{\varphi+1}}{\mathcal{F}_{\rho, \varphi+1}^{\sigma}\left[\mathcal{\omega}\left(e_{2}^{\wp}-e_{1}^{\wp}\right)^{\rho}\right]}\left\{\begin{array}{c}
\left(\int_{0}^{1}\left|\ell^{\varphi}-\alpha\right| \mathcal{F}_{\rho, \varphi+1}^{\sigma}\left[\omega\left(e_{2}^{\wp}-e_{1}^{\wp}\right)^{\rho}\left(\ell^{\rho}-\alpha\right)\right]\left(\ell x^{\wp}+(1-\ell) e_{2}^{\wp}\right)^{\frac{s}{\xi}-s} d \ell\right)^{\frac{1}{s}} \\
\times\left(\int_{0}^{1}\left|\ell^{\varphi}-\alpha\right| \mathcal{F}_{\rho, \varphi+1}^{\sigma}\left[\mathcal{\omega}\left(e_{2}^{\wp}-e_{1}^{\wp}\right)^{\rho}\left(\ell^{\rho}-\alpha\right)\right]\left|F^{\prime}\left(\wp \sqrt{\ell x^{\wp}+(1-\ell) e_{2}^{\wp}}\right)\right|^{r} d \ell\right)^{\frac{1}{r}} .
\end{array}\right.
\end{aligned}
$$

Since $\left|F^{\prime}\right|^{r}$ is $\wp$-convex on $\Omega$, we have

$$
\begin{gathered}
\left|\mathrm{Y}_{F}\left(\wp ; \alpha, \varphi, \rho, \sigma ; e_{1}, e_{2}\right)\right| \\
\leq \frac{\left(x^{\wp}-e_{1}^{\wp}\right)^{\varphi+1}}{\mathcal{F}_{\rho, \varphi+1}^{\sigma}\left[\omega\left(e_{2}^{\wp}-e_{1}^{\wp}\right)^{\rho}\right]}\left\{\begin{array}{c}
\left(\int_{0}^{1}\left|\ell^{\varphi}-\alpha\right| \mathcal{F}_{\rho, \varphi+1}^{\sigma}\left[\omega\left(e_{2}^{\wp}-e_{1}^{\wp}\right)^{\rho}\left(\ell^{\rho}-\alpha\right)\right]\left(\ell x^{\wp}+(1-\ell) e_{1}^{\wp}\right)^{\frac{s}{\zeta}-s} d \ell\right)^{\frac{1}{s}} \\
\times\left(\left|F^{\prime}(x)\right|^{r} \int_{0}^{1} \ell\left|\ell^{\varphi}-\alpha\right| \mathcal{F}_{\rho, \varphi+1}^{\sigma}\left[\omega\left(e_{2}^{\wp}-e_{1}^{\wp}\right)^{\rho}\left(\ell^{\rho}-\alpha\right)\right]\right. \\
\left.+\left|F^{\prime}\left(e_{1}\right)\right|^{r} \int_{0}^{1}(1-\ell)\left|\ell^{\varphi}-\alpha\right| \mathcal{F}_{\rho, \varphi+1}^{\sigma}\left[\omega\left(e_{2}^{\wp}-e_{1}^{\wp}\right)^{\rho}\left(\ell^{\rho}-\alpha\right)\right] d \ell\right)^{\frac{1}{r}}
\end{array}\right. \\
-\frac{\left(e_{2}^{\wp}-x^{\wp}\right)^{\varphi+1}}{\mathcal{F}_{\rho, \varphi+1}^{\sigma}\left[\omega\left(e_{2}^{\wp}-e_{1}^{\wp}\right)^{\rho}\right]}\left\{\begin{array}{c}
\left(\int_{0}^{1}\left|\ell^{\varphi}-\alpha\right| \mathcal{F}_{\rho, \varphi+1}^{\sigma}\left[\omega\left(e_{2}^{\wp}-e_{1}^{\wp}\right)^{\rho}\left(\ell^{\rho}-\alpha\right)\right]\left(\ell x^{\wp}+(1-\ell) e_{2}^{\wp}\right)^{\frac{s}{\varphi}-s} d \ell\right)^{\frac{1}{s}} \\
\times\left(\left|F^{\prime}(x)\right|^{r} \int_{0}^{1} \ell\left|\ell^{\varphi}-\alpha\right| \mathcal{F}_{\rho, \varphi+1}^{\sigma}\left[\omega\left(e_{2}^{\wp}-e_{1}^{\wp}\right)^{\rho}\left(\ell^{\rho}-\alpha\right)\right]\right. \\
\left.+\left|F^{\prime}\left(e_{2}\right)\right|^{r} \int_{0}^{1}(1-\ell)\left|\ell^{\varphi}-\alpha\right| \mathcal{F}_{\rho, \varphi+1}^{\sigma}\left[\omega\left(e_{2}^{\wp}-e_{1}^{\wp}\right)^{\rho}\left(\ell^{\rho}-\alpha\right)\right] d \ell\right)^{\frac{1}{r}} .
\end{array}\right.
\end{gathered}
$$

Therefore, we have

$$
\begin{aligned}
& \int_{0}^{1} \ell\left|\ell^{\varphi}-\alpha\right| \mathcal{F}_{\rho, \varphi+1}^{\sigma}\left[\omega\left(e_{2}^{\wp}-e_{1}^{\wp}\right)^{\rho}\left(\ell^{\rho}-\alpha\right)\right] d \ell \\
& \left.=\sum_{\kappa=0}^{\infty} \frac{\sigma(\kappa) \omega^{\kappa}\left(e_{2}^{\wp}-e_{1}^{\wp}\right)}{\Gamma(\rho \kappa+\varphi+1)}\right)\left[\frac{4 \alpha^{\frac{\varphi+\rho \kappa+2}{\varphi}}}{(\rho \kappa+2)(\rho \kappa+\varphi+2)}+\frac{1}{\rho \kappa+\varphi+2}-\frac{\alpha}{\rho \kappa+2}\right] .
\end{aligned}
$$

Analogously, we have

$$
\begin{aligned}
& \int_{0}^{1}(1-\ell)\left|\ell^{\varphi}-\alpha\right| \mathcal{F}_{\rho, \varphi+1}^{\sigma}\left[\omega\left(e_{2}^{\wp}-e_{1}^{\wp}\right)^{\rho}\left(\ell^{\rho}-\alpha\right)\right] d \ell \\
& =\sum_{\kappa=0}^{\infty} \frac{\sigma(\kappa) \omega^{\kappa}\left(e_{2}^{\wp}-e_{1}^{\wp}\right)}{\Gamma(\rho \kappa+\varphi+1)}\left[\frac{2 \varphi \alpha^{\frac{\varphi+\rho \kappa+1}{\varphi}}}{(\rho \kappa+1)(\rho \kappa+\varphi+1)}-\frac{4 \alpha^{\frac{\varphi+\rho \kappa+2}{\varphi}}}{(\rho \kappa+2)(\rho \kappa+\varphi+2)}\right. \\
& \left.\quad+\frac{1}{(\rho \kappa+\varphi+1)(\rho \kappa+\varphi+2)}-\frac{\alpha}{(\rho \kappa+2)(\rho \kappa+1)}\right] .
\end{aligned}
$$

Since $\wp \in\left(0, \frac{1}{1+\frac{1}{s}}\right]$, utilising Lemma 5 , we have that 


$$
\left(\ell x^{\wp}+(1-\ell) \beta^{\wp}\right)^{\frac{s}{\wp}-s} \leq 2^{\frac{s}{\wp}-s-1}\left(\ell^{\frac{s}{\wp}-s} x^{s-s \wp}+(1-\ell)^{\frac{s}{\wp}-s} \beta^{s-s \wp}\right), \quad \forall \ell \in[0,1], \beta \in\left\{e_{1}, e_{2}\right\} .
$$

It follows that

$$
\begin{aligned}
& \int_{0}^{1}\left|\ell^{\varphi}-\alpha\right| \mathcal{F}_{\rho, \varphi+1}^{\sigma}\left[\omega\left(e_{2}^{\wp}-e_{1}^{\wp}\right)^{\rho}\left(\ell^{\rho}-\alpha\right)\right]\left(\ell x^{\wp}+(1-\ell) \beta^{\wp}\right)^{\frac{s}{\wp}-s} d \ell \\
& \leq 2^{\frac{s}{\wp}-s-1} \int_{0}^{1}\left|\ell^{\varphi}-\alpha\right| \mathcal{F}_{\rho, \varphi+1}^{\sigma}\left[\omega\left(e_{2}^{\wp}-e_{1}^{\wp}\right)^{\rho}\left(\ell^{\rho}-\alpha\right)\right]\left(\ell^{\frac{s}{\wp}-s} x^{s-s \wp}+(1-\ell)^{\frac{s}{\wp}-s} \beta^{s-s \wp}\right) d \ell
\end{aligned}
$$

where

$$
\begin{aligned}
& \int_{0}^{1}\left|\ell^{\varphi}-\alpha\right| \mathcal{F}_{\rho, \varphi+1}^{\sigma}\left[\omega\left(e_{2}^{\wp}-e_{1}^{\wp}\right)^{\rho}\left(\ell^{\rho}-\alpha\right)\right]\left(\ell^{\frac{s}{\varsigma}-s} x^{s-s \wp}+(1-\ell)^{\frac{s}{\wp}-s} \beta^{s-s \wp}\right) d \ell \\
& =\sum_{\kappa=0}^{\infty} \frac{\sigma(\kappa) \omega^{\kappa}\left(e_{2}^{\wp}-e_{1}^{\wp}\right)}{\Gamma(\rho \kappa+\varphi+1)}\left[x ^ { s - s \wp } \left\{\frac{2 \alpha^{\frac{\wp \rho \kappa+s-s \wp+2 \wp}{\varphi}}}{\rho \kappa+\frac{s}{\wp}-s+1}-\frac{2 \alpha^{\frac{\wp \rho \kappa+\wp \varphi+s-s \wp+\wp}{\varphi}}}{\varphi+\rho \kappa+\frac{s}{\wp}-s+1}+\frac{1}{\varphi+\rho \kappa+\frac{s}{\wp}-s+1}\right.\right. \\
& \quad-\frac{\alpha}{\rho \kappa+\frac{s}{\wp}-s+1}+\beta^{s-s \wp}\left[\alpha\left(\mathbb{B}_{\alpha^{1 / \varphi}}(\rho \kappa+1, s / \wp-s+1)-\mathbb{B}_{1-\alpha^{1 / \varphi}}(\rho \kappa+1, s / \wp-s+1)\right)\right. \\
& \left.\left.\left.\quad-\mathbb{B}_{\alpha^{1 / \varphi}}(\varphi+\rho \kappa+1, s / \wp-s+1)+\mathbb{B}_{1-\alpha^{1 / \varphi}}(\varphi+\rho \kappa+1, s / \wp-s+1)\right)\right]\right\} .
\end{aligned}
$$

Combining (44) and (45), we get the desired inequality (46). So, this completes the proof.

To prove $(b)$, suppose that $\wp \in\left(\frac{1}{1+\frac{1}{s}}, 1\right]$, then we get the required inequality in (47) by using the inequality (14)

$$
\left(\ell x^{\wp}+(1-\ell) \beta^{\wp}\right)^{\frac{s}{\wp}-s} \leq\left(\ell^{\frac{s}{\wp}-s} x^{s-s \wp}+(1-\ell)^{\frac{s}{\wp}-s} \beta^{s-s \wp}\right), \quad \forall \ell \in[0,1], \beta \in\left\{e_{1}, e_{2}\right\}
$$

So, this completes the proof.

Corollary 4. Theorem 4 with $\left|F^{\prime}\right| \leq \mathcal{M}$ reduces to

(a) For $\wp \in\left(0, \frac{1}{1+\frac{1}{s}}\right]$, the following inequality holds:

$$
\begin{aligned}
& \left|\mathrm{Y}_{F}\left(\wp ; \alpha, \varphi, \rho, \sigma ; e_{1}, e_{2}\right)\right| \\
& \leq 2^{\frac{s}{\varsigma}-s-1} \mathcal{M}\left(\Phi_{1}(r)+\Phi_{2}(r)\right)^{\frac{1}{r}}\left[\frac{\left(x^{\wp}-e_{1}^{\wp}\right)^{\varphi+1} \Psi^{\frac{1}{s}}\left(e_{1}\right)+\left(e_{2}^{\wp}-x^{\wp}\right)^{\varphi+1} \Psi^{\frac{1}{s}}\left(e_{2}\right)}{\mathcal{F}_{\rho, \varphi+1}^{\sigma}\left[\omega\left(e_{2}^{\wp}-e_{1}^{\wp}\right)^{\rho}\right]}\right] .
\end{aligned}
$$

(b) For $\wp \in\left(\frac{1}{1+\frac{1}{s+1}}, 1\right]$, the following inequality holds:

$$
\begin{aligned}
& \left|\mathrm{Y}_{F}\left(\wp ; \alpha, \varphi, \rho, \sigma ; e_{1}, e_{2}\right)\right| \\
& \leq \mathcal{M}\left(\Phi_{1}(r)+\Phi_{2}(r)\right)^{\frac{1}{r}}\left[\frac{\left(x^{\wp}-e_{1}^{\wp}\right)^{\varphi+1} \Psi^{\frac{1}{s}}\left(e_{1}\right)+\left(e_{2}^{\wp}-x^{\wp}\right)^{\varphi+1} \Psi^{\frac{1}{s}}\left(e_{2}\right)}{\mathcal{F}_{\rho, \varphi+1}^{\sigma}\left[\omega\left(e_{2}^{\wp}-e_{1}^{\wp}\right)^{\rho}\right]}\right] .
\end{aligned}
$$




\section{Applications}

\subsection{Matrices}

Consider $\mathbb{C}^{n}$ represents the set of $n \times n$ completes matrices and $\mathbb{M}_{n}$ denotes the algebra of $n \times n$ complex matrices and $\mathbb{M}_{n}^{+}$be the strictly positive matrices in $\mathbb{M}$, i.e., $G \in \mathbb{M}_{n}^{+}$if $\langle G x, x\rangle>0$ for all nonzero $x \in \mathbb{C}^{n}$.

In [37], author derived the formula

$$
F(w)=\left\|G^{w} Y H^{1-w}+G^{1-w} Y H^{w}\right\|, \quad G, H \in \mathbb{M}_{n}^{+}, Y \in \mathbb{M}_{n}
$$

is convex for all $w \in[0,1]$. Then, this non-negative function is $\wp$-convex on $[0,1]$. Then, by Theorem 1 having $G, H \in \mathbb{M}_{n}^{+}, Y \in \mathbb{M}_{n}$, respectively, we have

Proposition 1. Let $G, H \in \mathbb{M}_{n}^{+}, Y \in \mathbb{M}_{n}$. Then one has

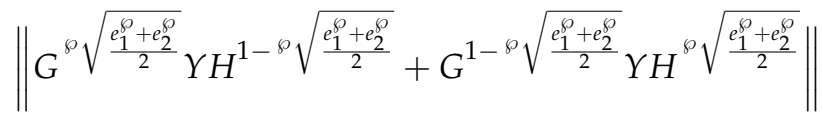

$$
\begin{aligned}
& \leq \frac{1}{2\left(e_{2}^{\wp}-e_{1}^{\wp}\right)^{\varphi} \mathcal{F}_{\rho, \varphi+1}^{\sigma}\left(\omega\left(e_{2}^{\wp}-e_{1}^{\wp}\right)^{\rho}\right)} \\
& \times\left\{\mathcal{J}_{\rho, \varphi,\left(e_{1}^{\wp}\right)^{+} ; \omega}^{\sigma}\left\|G^{\wp} \sqrt{e_{2}} \Upsilon H^{1-\wp} \sqrt{e_{2}}+G^{1-\wp \sqrt{e_{2}}} \Upsilon H^{\wp} \sqrt{e_{2}}\right\|+\mathcal{J}_{\rho, \varphi,\left(e_{2}^{\wp}\right)^{-} ; \omega}^{\sigma}\left\|G^{\wp} \sqrt{e_{1}} \Upsilon H^{1-\wp \sqrt{e_{1}}}+G^{1-\wp} \sqrt{e_{1}} Y H^{\wp} \sqrt{e_{1}}\right\|\right\} \\
& \leq \frac{1}{2}\left[\left\|G^{e_{1}} Y H^{1-e_{1}}+G^{1-e_{1}} Y H^{e_{1}}\right\|+\left\|G^{e_{2}} Y H^{1-e_{2}}+G^{1-e_{2}} Y H^{e_{2}}\right\|\right] \text {. }
\end{aligned}
$$

Proof. Let $F(w)$ is $\wp$-convex on $[0,1]$. Then the desired inequality (49) can be derived by applying inequality (15) to the mapping (48).

\subsection{Fox-Wright Function}

Let us take

$$
\sigma(\kappa)=\frac{\Gamma(\rho+\kappa)}{\kappa !} \frac{\prod_{i=1}^{p} \Gamma\left(a_{i}+\delta_{i} \kappa\right)}{\prod_{j=1}^{q} \Gamma\left(b_{j}+\gamma_{j} \kappa\right)} .
$$

In (3), then Raina's function becomes the Fox-Wright function proposed by (see, [38])

$$
\left.p \mathrm{Y}_{q}[x] \equiv p \mathrm{Y}_{q}\left[\begin{array}{l}
\left(a_{i}, \delta_{i}\right)_{1, p} \\
\left(b_{j}, \gamma_{j}\right)_{1, p}
\end{array}\right] x\right]:=\sum_{\kappa=0}^{\infty} \frac{\prod_{i=1}^{p} \Gamma\left(a_{i}+\delta_{i} \kappa\right) x^{\kappa}}{\prod_{j=1}^{q} \Gamma\left(b_{j}+\gamma_{j} \kappa\right) \kappa !},
$$

for all $x, a_{i}, b_{j} \in \mathbb{C}, \delta_{i}, \gamma_{j}(i=1,2, \ldots, p, j=1,2, \ldots, q)$,

$$
\Delta:=\sum_{j=1}^{q} \gamma_{j}-\sum_{i=1}^{p} \delta_{i}
$$

where the identity in the convergence condition holds true for appropriate bounded values of $|x|$ stated as

$$
|x|<\eta:=\frac{\prod_{j=1}^{q}\left|\gamma_{j}\right|^{\gamma_{j}}}{\prod_{i=1}^{p}\left|\delta_{i}\right|^{\delta_{i}}}
$$


and $|x|=\eta$, Then

$$
\varsigma:=\sum_{j=1}^{q} b_{j}-\sum_{i=1}^{p} a_{i}+\frac{p-q}{2}>\frac{1}{2} .
$$

Therefore, the left and right sided fractional integral operators derived from (4) and (5) are presented by

$$
\left(U_{\omega, e_{1}^{+} ;\left(b_{q}, \gamma_{q}\right)}^{\varphi, \rho ;\left(a_{p}, \delta_{p}\right)} F\right)(x)=\int_{e_{1}}^{x}(x-\ell)^{\varphi-1}{ }_{p} \mathrm{Y}_{q}\left[\omega(x-\ell)^{\rho}\right] F(\ell) d \ell
$$

and

$$
\left(U_{\omega, e_{2}^{-} ;\left(b_{q}, \gamma_{q}\right)}^{\varphi, \rho\left(a_{p}, \delta_{p}\right)} F\right)(x)=\int_{x}^{e_{2}}(\ell-x)^{\varphi-1}{ }_{p} \mathrm{Y}_{q}\left[\omega(\ell-x)^{\rho}\right] F(\ell) d \ell .
$$

In addition, we have

$$
\begin{aligned}
\mathcal{F}_{\rho, \varphi+1}^{\sigma}\left[\mathcal{\omega}(z-y)^{\rho}\right] & =\sum_{\kappa=0}^{\infty} \frac{\Gamma(\rho \kappa+\varphi)}{\Gamma(\rho \kappa+\varphi+1)} \frac{\prod_{i=1}^{p} \Gamma\left(a_{i}+\delta_{i} \kappa\right) \omega^{\kappa}(z-y)^{\rho \kappa}}{\prod_{j=1}^{q} \Gamma\left(b_{j}+\gamma_{j} \kappa\right) \kappa !} \\
& ={ }_{p+1} Y_{q+1}\left[\begin{array}{c}
(\varphi, \rho),\left(a_{i}, \delta_{i}\right)_{1, p} \\
; \omega(z-y)^{\rho} \\
(\varphi+1, \rho),\left(b_{j}, \gamma_{j}\right)_{1, q}
\end{array}\right] \\
& ={ }_{p+1} Y_{q+1}\left[\omega(z-y)^{\rho}\right] .
\end{aligned}
$$

Proposition 2. Let $0<e_{1}<e_{2}$ and $\alpha \in[0,1]$. Then

(a) For $\wp \in\left(0, \frac{1}{2}\right]$, the following inequality holds:

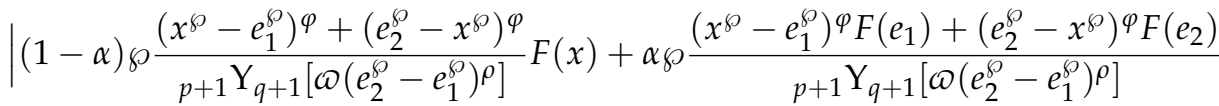

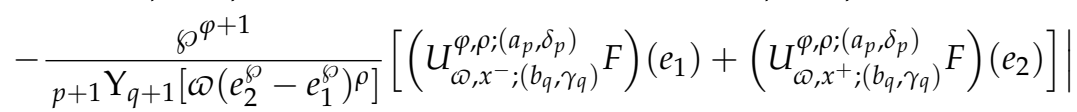

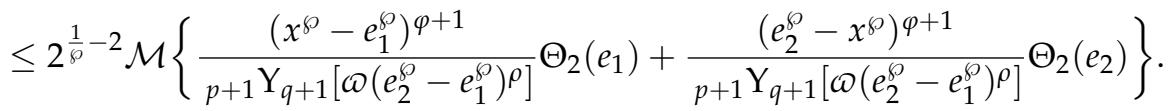

(b) For $\wp \in\left(\frac{1}{2}, 1\right]$, the following inequality holds:

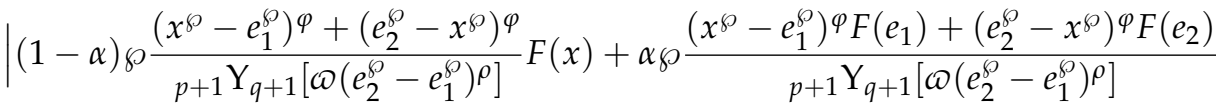

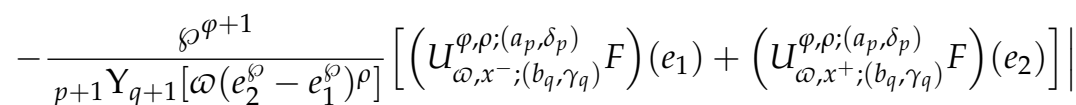

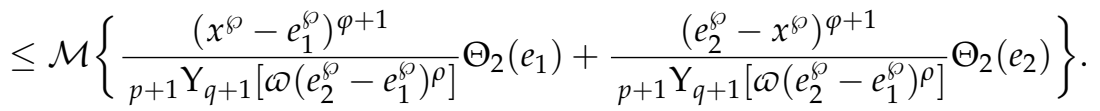

where $\Theta_{1}(\beta)$ and $\Theta_{2}(\beta)$ are defined by (29) and (30), respectively.

Proof. From (52) and (53), we know that the function ${ }_{p+1} \mathrm{Y}_{q+1}\left[\omega(z-y)^{\rho}\right]$ is $\wp$-convex on $\left[e_{1}, e_{2}\right]$. Therefore, inequality (55) and (56) can be derived by Theorem 2 immediately. 


\section{Conclusions}

The fractional integral inequalities for $\wp$-convex functions in the sense of generalised fractional integral operator have been successfully derived in this article. Here, we concentrate on all derived results in the current study that have been sustained for classical harmonically and classical convex functions, which can be obtained by letting $\wp=-1$ or 1 . All the derived outcomes are supported by applications in matrices and Fox-Wright function to relate and validate them. Consequently, this investigation shed light on special functions and existing fractional integral operators. Recently, author [39] has pondered Hermite-Hadamard's inequality on higher dimensions. Furthermore, it will be an appealing problem for ongoing research to analyse the outcomes achieved in this paper on higher dimensions. Therefore, this fascinating topic of research stimulates all other researchers to work on further investigation of $n$-polynomial $\wp$-convexity defined in other fractional operators.

Author Contributions: Conceptualisation, S.R., A.K., O.B. and G.I.O.; methodology, S.R., A.K., O.B. and G.I.O.; investigation, S.R., A.K., O.B., G.I.O.; resources, S.R., A.K., O.B. and G.I.O.; data curation, S.R., A.K., O.B., G.I.O.; writing—original draft preparation, S.R., A.K., O.B. and G.I.O.; writing-review and editing, S.R., A.K., O.B. and G.I.O.; supervision, S.R., A.K., O.B., G.I.O.; project administration, S.R., A.K., O.B., G.I.O.; funding acquisition, S.R., A.K., O.B., G.I.O. All authors read and agreed to the published version of the manuscript.

Funding: This research received no external funding.

Institutional Review Board Statement: Not applicable.

Data Availability Statement: Not applicable.

Acknowledgments: This research was supported by Taif University Researchers Supporting Project Number (TURSP-2020/96), Taif University, Taif, Saudi Arabia.

Conflicts of Interest: The authors declare no conflict of interest.

\section{References}

1. AlGhamdi, A.; Bazighifan, O.; El-Nabulsi, R.A. Important criteria for asympotatic properties of nonlinear differential equations. Mathematics 2021, 9, 1659. [CrossRef]

2. Bazighifan, O.; Almutairi, A.; Almarri, B.; Marin, M. An oscillation criterion of nonlinear differential equations with advanced term. Symmetry 2021, 13, 843. [CrossRef]

3. Abdeljawad, T.; Baleanu, D. Monotonicity results for fractional difference operators with discrete exponential kernels. Adv. Differ. Equ. 2017, 2017 , 78. [CrossRef]

4. Dokuyucu, M.A. A fractional order alcoholism model via Caputo-Fabrizio derivative. AIMS Math. 2019. 5, 781-797. [CrossRef]

5. Althubiti, S.; Bazighifan, O.; Alotaibi, H.; Awrejcewicz, J. New oscillation criteria for neutral deley differential equations of fourth-order. Symmetry 2021, 13, 1277. [CrossRef]

6. Rashid, S.; Khalid, A.; Sultana, S.; Hammouch, Z.; Shah, R.; Alsharif, A.M. A novel analytical view of time-fractional Korteweg-De Vries equations via a new integral transform. Symmetry 2021, 13, 1254. [CrossRef]

7. Verma, P.; Kumar, M.; Shukla, A. Analysis on Krasnoselskii's fixed point theorem of fuzzy variable fractional differential equation for a novel coronavirus (COVID-19) model with singular operator. Inter. J. Model. Sim. Sci. Comput. 2021, 12, 2150034. [CrossRef]

8. Atkinson, C.; Osseiran, A. Rational solutions for the time-fractional diffusion Eeuation. SIAM J. Appl. Math. 2021, 71, 92-106. [CrossRef]

9. Liang, Y.; Wang, S.; Chen, W.; Zhou, Z.; Magin, R.L. A survey of models of ultraslow diffusion in heterogeneous materials. Appl. Mech. Rev. 2019, 71, 040802. [CrossRef]

10. Rashid, S.; Ashraf, R.; Nisar, K.S.; Abdeljawad, T. Estimation of integral inequalities using the generalized fractional derivative operator in the Hilfer sense. J. Math. 2020, 2020, 1626091. [CrossRef]

11. Ge-JiLe, H.; Rashid, S.; Noor, M.A.; Suhail, A.; Chu, Y.-M. Some unified bounds for exponentially TGS-convex functions governed by conformable fractional operators. AIMS Math. 2020, 5, 6108-6123. [CrossRef]

12. Abdeljawad, T.; Rashid, S.; Hammouch, Z.; Chu, Y.-M. Some new local fractional inequalities associated with generalized $(s, m)$-convex functions and applications. Adv. Diff. Eq. 2020, 2020, 406. [CrossRef]

13. Abdeljawad, T.; Rashid, S.; Khan, H.; Chu, Y.-M. On new fractional integral inequalities for p-convexity within interval-valued functions. Adv. Diff. Eq. 2020, 2020, 330. [CrossRef]

14. Rashid, S.; Sultana, S.; Hammouch, Z.; Jarad, F.; Hamed, Y.S. Novel aspects of discrete dynamical type inequalities within fractional operators having generalized h-discrete Mittag-Leffler kernel. Chaos Solitons Fract. 2021, 151. [CrossRef] 
15. Zhou, S.-S.; Rashid, S.; Rauf, A.; Jarad, F.; Hamed, Y.S.; Abualnaja, K.M. Efficient computations for weighted generalized proportional fractional operators with respect to a monotone function. AIMS Math. 2021, 6, 8001-8029. [CrossRef]

16. Ostrowski, A. Über die Absolutabweichung einer differentiierbaren Funktion von ihrem Integralmittelwert. Comment. Math. Helv. 1937, 10, 226-227. [CrossRef]

17. Rashid, S.; Sultana, S.; Jarad, F.; Jafari, H.; Hamed, Y.S. More efficient estimates via h-discrete fractional calculus theory and applications. Chaos Solitons Fract. 2021, 147, 110981. [CrossRef]

18. Ge-Jile, H.; Rashid, S.; Farooq, F.B.; Sultana, S. Some inequalities for a new class of convex functions with applications via local fractional integral. J. Fun. Spaces. 2021, 2021, 6663971. [CrossRef]

19. Zhou, S.-S.; Rashid, S.; Parveen, S.; Akdemir, A.O.; Hammouch, Z. New computations for extended weighted functionals within the Hilfer generalized proportional fractional integral operators. AIMS Math. 2021, 6, 4507-4525. [CrossRef]

20. Rashid, S.; Chu, Y.-M.; Singh, J.; Kumar, D. A unifying computational framework for novel estimates involving discrete fractional calculus approaches. Alexandria Eng. J. 2021, 60, doi:10.1016/j.aej.2021.01.003 [CrossRef]

21. Rashid, S.; Hammouch, Z.; Ashraf, R.; Chu, Y.-M. New computation of unified bounds via a more general fractional operator using generalized Mittag-Leffler function in the kernel. Comput. Model. Eng. Sci. 2020, 126, 359-378.

22. Chu, Y.-M.; Rashid, S.; Abdeljawad, T.; Khalid, A.; Kalsoom, H. On new generalized unified bounds via generalized exponentially harmonically s-convex functions on fractal sets. Adv. Diff. Eq. 2021, 2021, 218. [CrossRef]

23. İşcan, İ. Ostrowski type inequalities for $p$-convex functions. New Trends. Math. Sci. 2016, 4, 140-150. [CrossRef]

24. Abdeljawad, T.; Rashid, S.; Hammouch, Z.; İşcan, İ.; Chu, Y.-M. Some new Simpson-type inequalities for generalized p-convex function on fractal sets with applications. Adv. Diff. Eq. 2020, 2020, 496. [CrossRef]

25. Chen, S.-B.; Rashid, S.; Noor, M.A.; Hammouch, Z.; Chu, Y.-M. New fractional approaches for n-polynomial P-convexity with applications in special function theory. Adv. Diff. Eq. 2020, 2020, 543. [CrossRef]

26. İşcan, İ.; Turhan, S.; Maden, S. Hermite-Hadamard and Simpson-like type inequalities for differentiable p-quasi-convex functions. Filomat 2017, 31, 5945-5953. [CrossRef]

27. Yu, Y.; Lei, H.; Hu, G.; Du, T. Estimates of upper bound for differentiable mappings related to Katugampola fractional integrals and $p$-convex mappings. AIMS Math. 20201, 6, 3525-3545. [CrossRef]

28. Raina, R.K. On generalized Wright's hypergeometric functions and fractional calculus operators. East Asian Math. J. 2005, 21, 191-203.

29. Alomari, M.; Darus, M.; Dragomir, S.S.; Cerone, P. Ostrowski type inequalities for functions whose derivatives are s-convex in the second sense. Appl. Math. Lett. 2010, 23, 1071-1076. [CrossRef]

30. Set, E. New inequalities of Ostrowski type for mappings whose derivatives are s-convex in the second sense via fractional integrals. Comput. Math. Appl. 2012, 63, 1147-1154. [CrossRef]

31. Thatsatian, A.; Ntouyas, S.K.; Tariboon, J. Some Ostrowski type inequalities for p-convex functions via generalized fractional integrals. J. Math. Inequal. 2019, 13, 467-478. [CrossRef]

32. Gürbüz, M.; Taşdan, Y.; Set, E. Some inequalities obtained by fractional integrals of positive real orders. J. Inequal. Appl. 2020, 152, 1-11. [CrossRef]

33. Rashid, S.; Noor, M.A.; Noor, K.I.; Chu, Y.-M. Ostrowski type inequalities in the sense of generalized K-fractional integral operator for exponentially convex functions. AIMS Math. 2020, 5, 2629-2645. [CrossRef]

34. Wang, J.; Deng, J.; Fečkan, M. Hermite-Hadamard-type inequalities for r-convex functions based on the use of Riemann-Liouville fractional integrals. Ukr. Math. J. 2013, 65, 193-211. [CrossRef]

35. İşcan, İ. Hermite-Hadamard type inequalities for harmonically convex functions. Hacet. J. Math. Stat. 2014, 43, 935-942. [CrossRef]

36. Dragomir, S.S.; Agarwal, R.P.; Barnett, N.S. Inequalities for Beta and Gamma functions via some classical and new integral inequalities. J. Inequal. Appl. 2000, 5, 103-165. [CrossRef]

37. Sababheh, M. Convex functions and means of matrices. arXiv 2016, arXiv:1606.08099.

38. Kilbas, A.A.; Srivastava, H.M.; Trujillo, J.J. Theory of Applications of Fractional Differentia Equations; Elesvier: Amsterdam, The Netherlands, 2006; Volume 204.

39. Steinerberger, S. The Hermite-Hadamard inequality in higher dimensions. J. Geo. Anal. 2020, 30, 466-483. [CrossRef] 